\title{
dspace.vutbr.cz
}

\section{An Experimental Analysis of the Spraying Processes in Improved Design of Effervescent Atomizer}

\author{
ZAREMBA, M.; KOZÁK, J.; MALÝ, M.; WEISS, L.; RUDOLF, P.; JEDELSKÝ, J.; JÍCHA, M.
}

International Journal of Multiphase Flow

2018, vol. 103, June 2018, pp. 1-15

ISSN: 0301-9322

DOI: https://doi.org/10.1016/j.ijmultiphaseflow.2018.01.012

Accepted manuscript

(C) 2018. This manuscript version is made available under the CC-BY-NC-ND 4.0 license (http://creativecommons.org/licenses/by-nc-nd/4.0/), doi:

https://doi.org/10.1016/j.jmultiphaseflow.2018.01.012

Final version available from https://www.sciencedirect.com/science/article/pii/s030193221730246X 


\section{An Experimental Analysis of the Spraying Processes in Improved Design of Effervescent Atomizer}

\section{Abstract}

This work experimentally examines the primary atomization processes in a newly developed atomizer, similar to effervescent atomizer concept, at low pressures and low gas-to-liquid ratios (GLR). Several experimental and post-processing techniques are applied to investigate the spray spatial evolution. The near-nozzle area is captured by a high-speed camera with a long-distance microscope. Further, characteristics of the developed spray are investigated by a phase-Doppler analyser (PDA). The high-speed recordings are processed by the proper orthogonal decomposition (POD). The frequency analysis of examined phenomenon is done by the fast Fourier transformation (FFT) at selected positions in the images. The POD enables to sort out data according to the importance of characteristic shapes occurring in the recordings. The velocity and dimensions of discharging liquid are measured in images by a point-tracking method. Dimensionless criteria are estimated to describe the atomization principles where several new findings are found comparing the previous studies. The spatial spray evolution is described by the processed PDA data. A simplification, based on the Stokes number, is used to estimate a gas motion in the spray. This approach enables to investigate the interaction between the spray and ambient atmosphere. The combination of experimental and post-processing techniques confirms the previous findings of the improved effervescent atomizer. In other words, the atomizer operates inherently at annular twophase flow regime which, however, leads to a specific atomizing mechanism i.e. bubble bursts, the same as in the effervescent spraying process. However, an importance of the interaction between the two following bubble bursts is highlighted as driving atomization mechanism. This specific behaviour is reason why the atomizer can be operated at low consumption of gas and low-pressure regimes. Moreover, the applied experimental and post-processing techniques indicate a potential for further advanced data post-processing of the stochastic processes of liquid atomization.

\section{Introduction}

In fluid mechanics, especially sprays applications, atomization is understood as a fragmentation i.e. breakup of liquid into individual droplets. In industrial applications the spray character has a demonstrable effect on the process efficiency and product quality such as liquid fuel combustion technology, liquid jet propulsion, atomization of melts, agricultural sewage and irrigation, powder technology, ink-jet printing and others (Eggers and Villermaux 2008). Even though there is an increasing amount of renewable power sources, still of particular interest to the power generation is injection of a liquid fuel into combustors, incinerators and combustion chambers of engines. Nowadays, a trend in industry is to use alternative, biologically derived, hydrocarbon fuels with physical properties different from the commonly used ones. Thus, an extension of knowledge is needed to understand and optimize liquid atomization mechanisms and to develop or improve atomizers to new applications and higher demands on the spray quality. 
Atomizer designs are extensively studied, and several new concepts were developed in the past decades such as electro static atomizer, internally-mixed twin-fluid atomizer (Ashgriz 2011) and designs combining different atomizing techniques (Kourmatzis, Lowe, and Masri 2016) and others. In the present paper, an internally-mixed twin-fluid atomizer based on the construction of a classical effervescent atomizer is investigated.

The effervescent atomizer was developed and studied by (Lefebvre 1988) and it has been intensively examined during last decades. The findings were summarized in review papers by (Sovani, Sojka, and Lefebvre 2001, Konstantinov et al. 2010, Qian and Lin 2011). The main advantages of effervescent atomizers are large exit orifices, low operating pressures, low consumption of gas comparing other twin-fluid atomizers and relative insensitivity to the liquid viscosity (Sovani, Sojka, and Lefebvre 2001). These characteristics were attributed to the breakup process which occurs during the effervescent atomization, the so-called bubble explosions (Konstantinov et al. 2010). Due to this specific liquid breakup mechanism, effervescent atomizers can operate at low operating pressures and generate a spray of considerably small mean drop size. Moreover, regimes characterized by the low operating pressure and low GLR are assumed as comparably highly effective (Jedelsky and Jicha 2013, Stähle, Schuchmann, and Gaukel 2015). However, the spray instabilities at these regimes are the main drawback of effervescent atomizers.

Even though the effervescent atomization is assumed as inherently unstable (Jedelsky and Jicha 2008; Luong et al., 1999), the spray may also become unstable from another point of view, e.g. more atomizing principles can be observed. It was shown that the flow discharge might change randomly from bubbly to annular flow undisturbed liquid column (Zaremba et al. 2017). Multiple breakup mechanisms usually cause a very poor atomization. This occurs mainly at relatively low flow rates (Zaremba et al. 2017, Gadgil and Raghunandan 2011, Mlkvik et al. 2015). An unsteady operation is caused by pulses, generated by explosions of gas bubbles, which enhances instability in the spray (Jedelsky and Jicha 2008, Luong and Sojka 1999). However, recent studies show that when a certain design is used, an annular flow can be created inside the atomizer for a broad range of operating conditions and GLRs. It improves the spray stability under low-pressure regimes as it was shown by (Mlkvik et al. 2015, Stähle, Schuchmann, and Gaukel 2015, Zaremba et al. 2017); therefore, a similar design is examined in the present paper to describe the primary atomization process of the improved effervescent atomizer under low-pressure and low-GLR regimes.

In the internally-mixed atomizers, such as the effervescent type, the gas and liquid phases enter the atomizer separately. The fluids interact inside the mixing chamber while creating a two-phase flow. The effervescent atomization is defined by the bubbly two-phase flow established inside the chamber (Sovani, Sojka, and Lefebvre 2001). The mixture then flows through the atomizer and when it passes the exit orifice, liquid is disrupted by the expanding gas. Primary atomization is a process which describes a breakup of initial liquid volume into its fractions, large structures and the so-called ligaments. These fractions then might break up into individual droplets, i.e. secondary atomization; further downstream from the atomizer.

The process of liquid atomization in the internally-mixed twin-fluid atomizers is strongly affected by the character of the internal flow. It was shown by (Whitlow et al. 1992, Chin 1995, Huang, Wang, and Liao 2008, Chin and Lefebvre 1993, Li et al. 2012) that the internal flow depends mainly on the gas-to-liquid ratio (GLR). When GLR is low, typically below $5 \%$, a bubbly flow occurs inside the effervescent atomizer. With an increase of GLR, the internal flow changes from the bubbly to plug flow. In the case of high GLRs, typically $10 \%$ and more, an annular two-phase flow is created inside the atomizer. The internal bubbly flow results in the breakup mechanism described as bubble explosions (Buckner and Sojka 1991, Gadgil and Raghunandan 2011). According to the experimental investigations of (Santangelo and Sojka 1995), the bubble explosions regime occurs at $G L R=2 \%$ and lower. When GLR increases to $5 \%$ and more, the breakup mechanism is described as the so-called 
tree-like regime (Sutherland, Sojka, and Plesniak 1997) which produces liquid structures in a shape of branched liquid fragments. This regime supports the simplification used in modelling of effervescent atomization where models predict an annular two-phase flow discharging from the orifice (Qian and Lin 2011). The findings presented in the above-mentioned articles describe the aspects of the effervescent atomization in general and they focus mainly on the experimental observation of the breakup mechanism with regard to the spray properties. Even the recent studies (Gadgil and Raghunandan 2011) do not describe the breakup process in a greater detail and the mechanism of the primary liquid deformation has not been described yet. Thus, a detailed description of the atomization process still needs to be given as pointed out by (Sovani, Sojka, and Lefebvre 2001, Konstantinov et al. 2010, Qian and Lin 2011). The present study is focused on the description of forces acting on the liquid during the discharge, initial deformation, primary breakup, with a link to the spatial spray evolution. For this purpose, a high-speed back illumination is used in the nearnozzle area to capture discharging liquid. Further downstream the phase Doppler anemometry (PDA) was used to analyse behaviour of droplets in the spray.

The analysis of breakup processes is a complex issue because of its stochastic nature. Several post-processing techniques were used to quantify visual experimental data such as fractal analysis (Dumouchel, Cousin, and Triballier 2005) or proper orthogonal decomposition (Chen et al. 2013). The POD technique was firstly introduced into the fluid mechanics by (Lumley 1967, Berkooz, Holmes, and Lumley 1993). Since then it has been used, for example, in the analysis of computation fluid dynamics and experimental data to describe the structures of rotating vortex rope in draft turbine (Rudolf, Štefan, and Klas 2015). In the spray research, it has been recently used for examination of cycle-to-cycle variations of the spray shape and penetration (Chen et al. 2013, Chen et al. 2014). The POD technique can transform the experimental or numerical data to the so-called modes which represent characteristic shapes occurring in the examined event. Therefore, the POD technique is used in this paper, as promising analysis tool, to obtain characteristics features of liquid during the primary atomization process from high-speed images.

This research describes the liquid breakup mechanism during effervescent atomization using a new design of twin-fluid atomizer at relatively high-efficient regimes. The results section provides a description of initial liquid deformation (primary atomization), and a further breakup of liquid fractions (secondary atomization). For this purpose, a high-speed back-illumination technique was used together with advanced image post-processing, the POD. Further examination of the entire spray was conducted by means of phase Doppler anemometry.

A combination of different experimental and analytical techniques gave us a base for a description of effervescent atomization. The collision of two following bursts, which enhances a breakup process, was highlighted as a main driving mechanism in breakup process. The results document several new features which have been found in comparison with the previous studies and show potential of applied experimental and post-processing techniques as a promising tool for studying of stochastic processes.

\section{Materials and Methods}

This section describes an experimental setup, test conditions of the atomizer, measuring and visualization techniques, and post-processing methods. The measurements were performed at an operating pressure of $70 \mathrm{kPa}$, GLRs: $2.5,5$ and $10 \%$, under atmospheric ambient conditions and at a room temperature. To control the GLR value during the measurement, a mass flow meter was used for liquid (combined uncertainty of measured value $1 \%$ for a confidence level of $95 \%$ ), mass flow meter for gas (5\%) and pressure sensors (0.4\%).

GLR is a ratio of mass flow rate of atomizing gas through the atomizer to that of liquid (Sovani et al. 2001). In this paper, it is expressed as a percentage of gas to liquid mass flow. 


\subsection{Test bench}

The atomizer was operated on the cold test bench, see Figure 1. The original application of this type of atomizer was spraying of viscous fuels. Thus, a light heating oil (LHO) and pressurized air were used as the test fluids. Physical properties of the fluids are defined in the Table 1.

Table 1. Physical properties of fluids at room temperature.

\begin{tabular}{|c|ccc|}
\hline Fluid & $\rho\left[\mathrm{kg} / \mathrm{m}^{3}\right]$ & $\mu[\mathrm{kg} /(\mathrm{m} \cdot \mathrm{s})]$ & $\sigma\left[\mathrm{kg} / \mathrm{s}^{2}\right]$ \\
\hline LHO & 874 & 0.0185 & 0.0297 \\
Air & 1.23 & $1.81 \cdot 10^{-5}$ & - \\
\hline
\end{tabular}

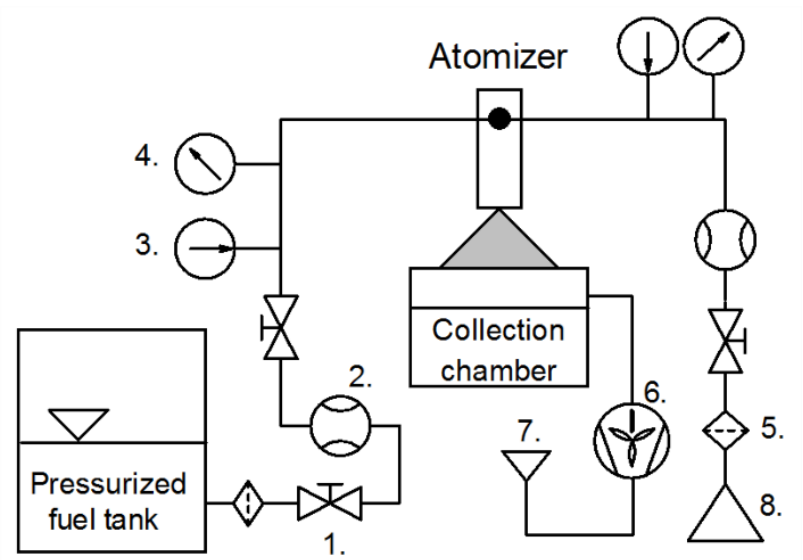

Fuel line

Air line

Figure 1 Schematic layout of the test bench: 1) Valve, 2) Flow meter, 3) Temperature sensor, 4) Pressure sensor, 5) Filter, 6) Mist filter, 7) Exhaust pipeline, 8) Central line of pressurized air.

The test bench contains two supply lines: fuel and air. Both the lines are equipped with pressure sensors, BD sensors DMP 33li, and thermometers, resistance temperature sensor Omega SPRTX-S1. The operating regimes of atomizer were controlled by pressure and mass flows of the fluids. The measured values of pressure and mass flow rates are documented in Table 2. LHO was supplied from the pressurized fuel tank through the filter and the control valve into the Siemens Mass 2100 Coriolis flow meter fitted with Mass 6000 Ex transmitter. The air was taken from the central pressurized line; it was flowing through the filter and the control valve into the Omega Mass Flow meter FMA A2117. The pressure and temperature sensors were placed behind the flow meters, at each line.

Table 2. Parameters of the operating regimes.

\begin{tabular}{|cccc|}
\hline $\begin{array}{c}p_{L} \\
{[\mathrm{kPa}]}\end{array}$ & $\begin{array}{c}p_{G} \\
{[\mathrm{kPa}]}\end{array}$ & $\begin{array}{c}m_{L} \\
{\left[\mathrm{~kg} \cdot \mathrm{h}^{-1}\right]}\end{array}$ & $\begin{array}{c}G L R \\
{[\%]}\end{array}$ \\
\hline 70 & 70 & 7.7 & 2.6 \\
70 & 69 & 5.6 & 5.0 \\
70 & 71 & 3.8 & 10.2 \\
\hline
\end{tabular}

\subsection{Atomizer}

The atomizer design is identical to that of the effervescent atomizer with one major difference liquid and gas inlets are inversely connected. It means that the gas flows through the centre of the atomizer, and the liquid is injected by a set of aerator holes into the mixing chamber. A similar construction was designed and used by (Stähle, Schuchmann, and Gaukel 2015) and (Mlkvik et al. 2015). The examined atomizer inherently creates an annular internal flow due to its construction, see Figure 2. Liquid is injected into the gas stream perpendicularly to the main axis. It remains on the walls of the mixing chamber due to its low momentum, which creates an annular internal two-phase 
flow. A gas-liquid mixture then flows through the atomizer; when it passes the exit orifice, the gas expands and shatters the liquid.

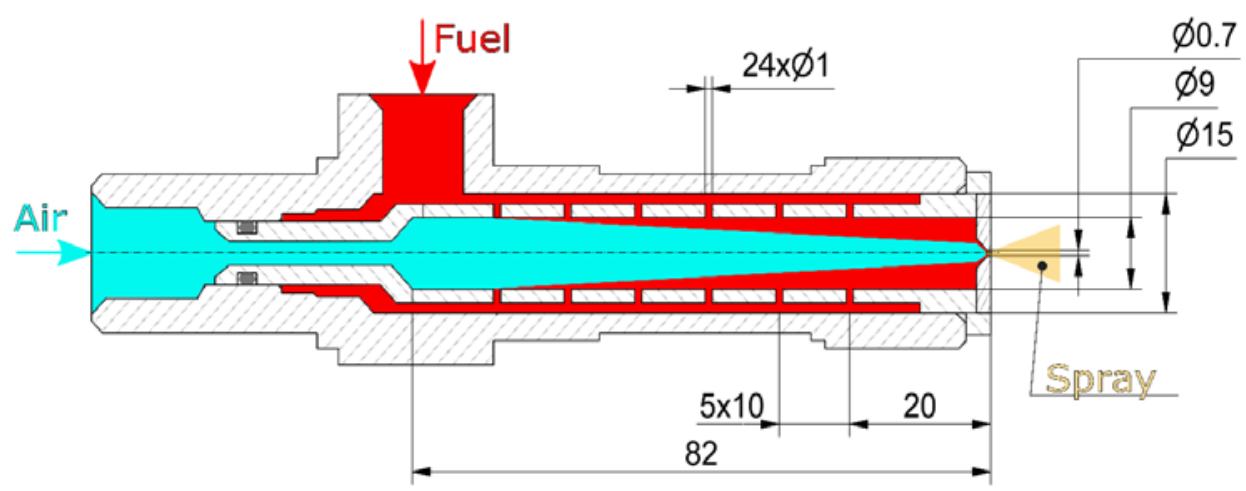

Figure 2 Schematic layout of the atomizer's function. During the tests the atomizer was mounted vertically and the spray flows downstream, see Figure 3.

\subsection{Measuring and visualization techniques}

A back-illumination technique was used for capturing an emerging two-phase flow in the near-nozzle area, see Figure 3. The area of interest was illuminated by the pulse laser Cavilux HF System $810 \mathrm{~nm} /$ $500 \mathrm{~W}$ with laser pulse duration of $50 \mathrm{~ns}$. To make the light intensity more homogenous, a light diffuser was placed in front of the laser. Recordings were done by the high-speed camera Photron SA-Z with long-distance microscope NAVITAR $12 \mathrm{X}$ with $0.25 \mathrm{X}$ attached lens and $1 \mathrm{X}$ F-mount adapter. The resulting resolutions of the images were $408 \times 384$ at frame-rate $100,000 \mathrm{fps}$, and $688 \times 1024$ pixels at frame rate $30,000 \mathrm{fps}$, which results in area dimensions of approximately $1.5 \times 1.4 \mathrm{~mm}$ and $12 \times 18 \mathrm{~mm}$ respectively. Two different resolutions were used to capture the primary and secondary atomization as each of these features requires a different dimensions of examined area.

A fibre based 2D phase-Doppler analyser by Dantec Dynamics was used to obtain the information on the droplet size and velocity, see Figure 3. This system simultaneously measures the droplet velocity in two directions, axial and radial, and its size. The PDA system consists of Spectra physics Stabilite 2017 Argon laser, 60X41 Transmitter which includes a beam splitter and a Bragg cell (phase shift of $40 \mathrm{MHz}$ ), 60X81 2D $85 \mathrm{~mm}$ transmitting optics with 50X82 beam translator, 57X50 $112 \mathrm{~mm}$ diameter receiving optics, fibre PDA detector unit and BSA P80 flow and a particle processor. Focal length of transmitting optics was $500 \mathrm{~mm}$ and for the receiving optics it was $800 \mathrm{~mm}$. The data were obtained from the point of laser beams intersection, the so-called measuring volume, which has an ellipsoid shape and dimensions of $0.6 \times 0.08 \times 0.08 \mathrm{~mm}$; the length, width and height respectively. A slit in the receiving optics was set to $0.2 \mathrm{~mm}$ to reduce the length of the measuring volume. The system was set to collect 30,000 samples or to measure for at least one minute in low-dense spray regions. Five axial distances were examined $(5,10,20,40,80 \mathrm{~mm})$; in each axial distance one radial profile was measured by 1 to $4 \mathrm{~mm}$ step depending on the width of the spray at a given axial position. The maximum measured droplet size was set to $166.54 \mu \mathrm{m}$ with size resolution of $\pm 0.05 \mu \mathrm{m}$, and the uncertainty of individual droplet size measurement was $\pm 0.5 \mu \mathrm{m}$. The axial and radial velocity range was set from $0-16 \mathrm{~m} / \mathrm{s}$ to $0-64 \mathrm{~m} / \mathrm{s}$ and from $0-64 \mathrm{~m} / \mathrm{s}$ to $0-128 \mathrm{~m} / \mathrm{s}$, respectively, considering the effect of the axial distance from the atomizer and the GLR on the maximum droplet velocity. The velocity resolution was $0.002 \%$, and the uncertainty was less than $1 \%$ of the selected range. 


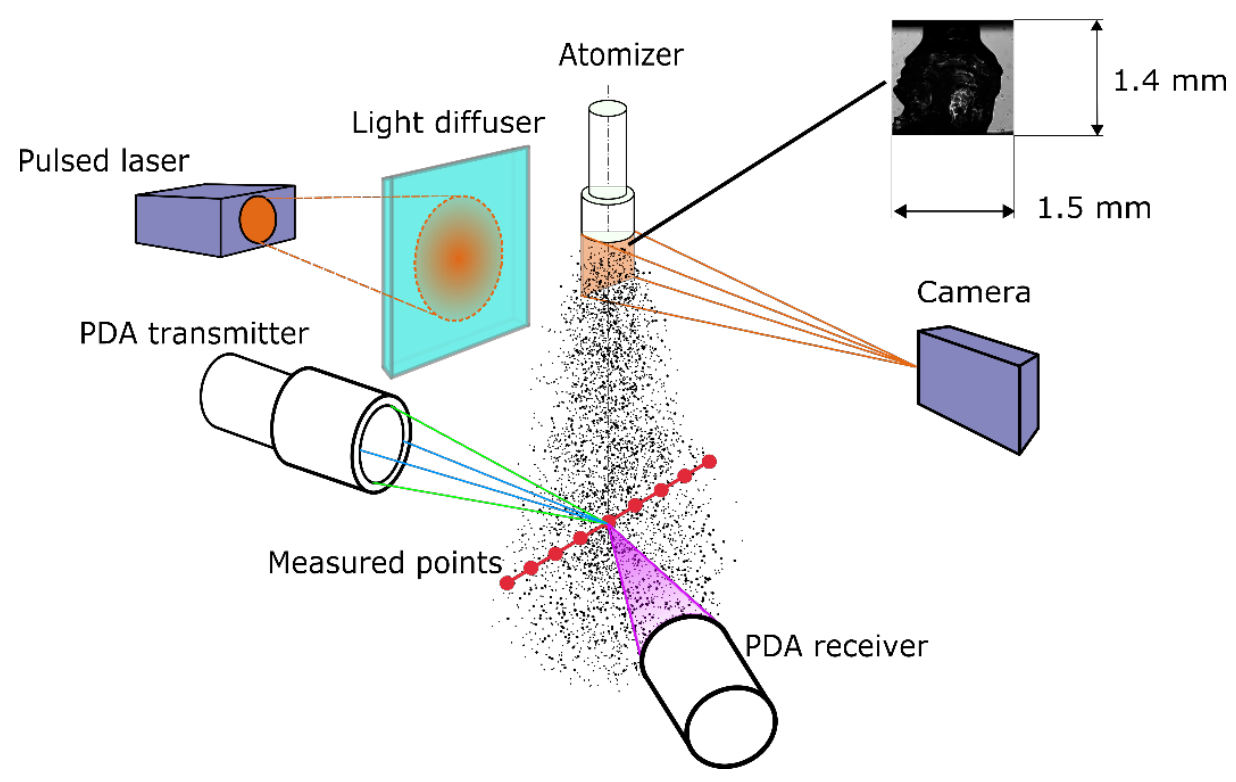

Figure 3 Schematic layout of the experimental setup (another image resolution of the images was $12 \times 18 \mathrm{~mm}$ ).

\subsection{Data analysis methods}

This section describes the post-processing methods used for data analysis. The image postprocessing was applied to the raw high-speed recordings. This serves for the description of the breakup mechanism and for the frequency analysis in the near-nozzle region. The PDA data was used for the analysis of the spray evolution further from the atomizer. In this section, we also introduce dimensionless numbers which give an insight into ratios of forces acting on the liquid during the spraying process.

\subsubsection{Image post-processing}

To get a quantitative information on the liquid deformation in the near-nozzle area root mean square images (RMS) were created from a sequence of snapshots (750 images), see Figure 4 b), in PCO Picture Viewer ${ }^{1}$. The analysis of visual data using RMS images was also performed in previous works such as (Marchi et al. 2010, Zaremba et al. 2017). The pixel intensities in the RMS image are computed by calculating the standard deviation of the pixel intensities of the image sequence. For better visibility of the pixel intensity, RMS figures were turned into the red-green-blue (RGB) colour spectrum, Figure $4 \mathrm{c}$ ). The same colour scale was then used for the representation of POD modes further in this paper.

The RMS images show differences of the pixel intensity among the examined sequences. Thus, a high intensity, red colour, indicates the areas of rapid liquid movements and vice versa. Moreover, the RMS images served for a global evaluation of the liquid breakup and confirmation of the frequency analysis as it is discussed in the following section. It should be noted that the examined problem of the liquid deformation is three-dimensional. Thus, the information in the images is distorted by the unequal optical density where light propagates from the light source to the camera. It means that the lowest light intensity appears in the core of the liquid where the light travels through the full diameter of the liquid column. In contrast, the information on the liquid edges corresponds to the real deformation of liquid, which is relevant under assumption of symmetry of examined phenomena.

\footnotetext{
${ }^{1}$ PCOPictures, DocSchneider Engineering, CH-8308 IIInau, Switzerland.
} 


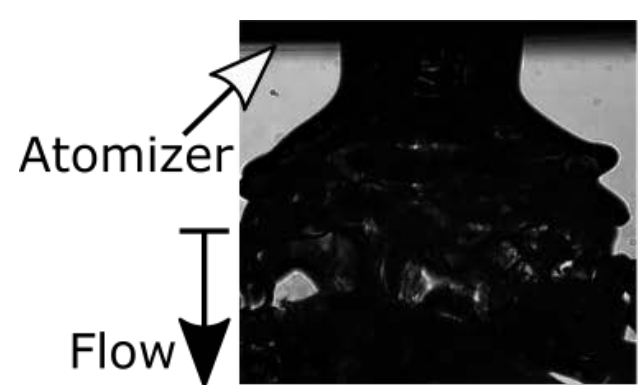
direction

a)

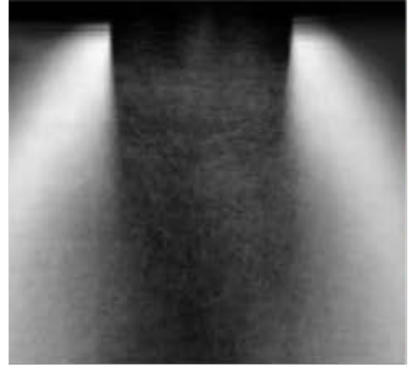

b)

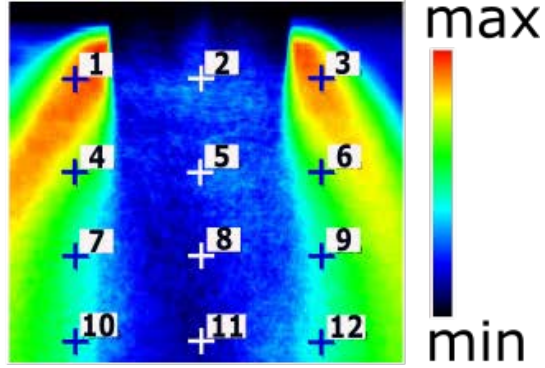

c)

Figure 4 Image post-processing technique: a) original snapshot, b) RMS image created from sequence of 750 snapshots, c) color-coded RMS image with highlighted points for the frequency analysis.

\subsubsection{Frequency analysis}

A frequency analysis, applying fast Fourier transformation (FFT), was used to get dominant frequencies of the liquid deformation, bubble expansion, and to support the results from the POD. Preliminary post-processing showed that the frequency which can be assumed as a dominant one was only found in the regions with the highest pixel intensity in RMS images, points 1 and 3, Figure 4 c). The remaining analysed points contained no distinguishable peak. A dominant frequency was partly visible in points 4 and 6 . In the Results section only the data from the point in the highest intensities in RMS image, point one, are analysed.

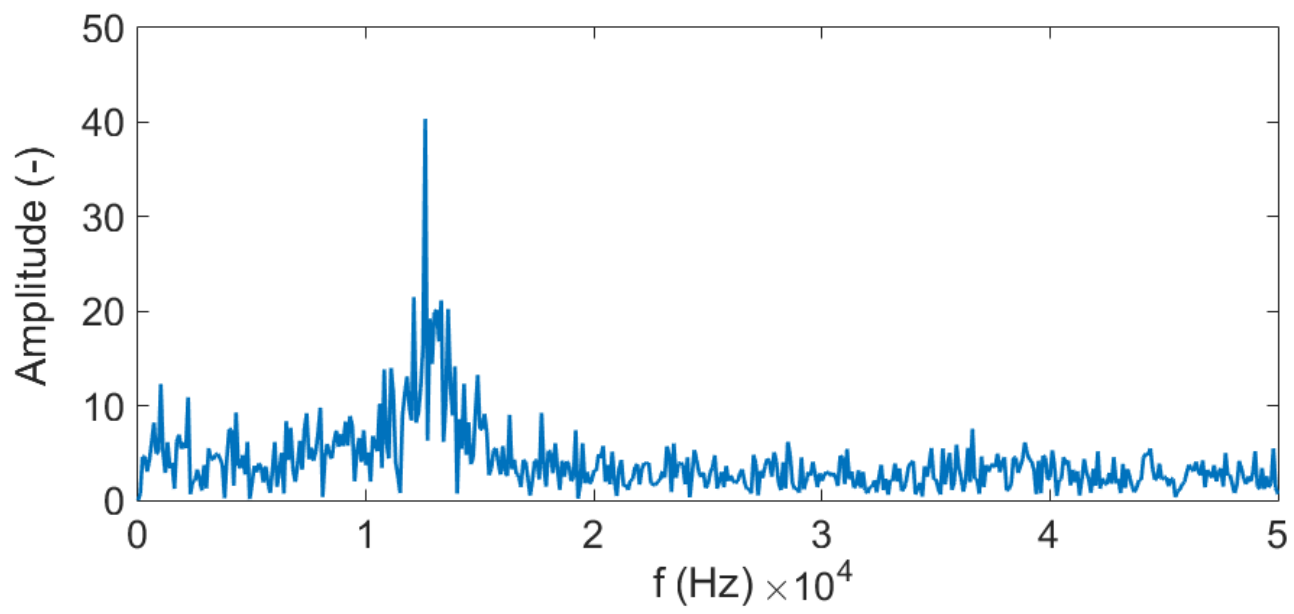

Figure 5 Single side amplitude spectrum of point 1, the frequency spectrum of point 1 in Fig. 4 c), which shows the dominant frequency of $12.6 \mathrm{kHz}$.

\subsubsection{Proper orthogonal decomposition}

This section gives an insight into the interpretation of the proper orthogonal decomposition and its modes. A POD technique was applied to the experimental data obtained by the back-illumination recordings. A detailed description of the POD technique can be found elsewhere in (Lumley 1967, Berkooz, Holmes, and Lumley 1993). In this paper, the description is limited to the following paragraphs:

POD enables to process numerical and experimental data and to quantitatively describe the main flow features. Mathematically, the objective of POD is to find a sequence of basis functions which represents the dominant structures from a given scalar field set (Chen et al. 2013). In other words, 
the result of the POD decomposition consists of the set of time and spatial coefficients representing the individual POD modes.

According to Lumley (1967), who tested POD on the velocity vector field, the individual POD modes represent a kinetic energy distribution. However, in the case of the experimental data examined in the present paper, the POD modes are not linked to a specific physical quantity, such as velocity or pressure, but to the variation of pixel intensity (e.g. the value between 0 and 255 of the greyscale) within the region of interest.

The use of POD is limited to the processing of the scalar pixel intensity of the back-illumination images. The time dependent eigenfunctions $a_{i}$ and spatio-dependent functions $\phi_{i}$ are computed in order to find an approximation of general spatio-temporal dependent variable $u(x ; t)$ (in this case pixel intensity I) within the domain $\Omega$. For this purpose, the correlation matrix $C_{i j}$ is constructed using the samples of the record with known sampling frequency according to the following equation:

$$
C_{i j}=\frac{1}{M} \int_{\Omega} u\left(\boldsymbol{x} ; t_{i}\right) u\left(\boldsymbol{x} ; t_{j}\right) d \boldsymbol{x}
$$

where $M$ is number of snapshots. Solving the eigenvalue problem of the correlation matrix $C_{i j}$, the eigenvalues and eigenvectors are obtained. Considering that the eigenvalue $\lambda$ is paired with corresponding eigenvector $e$ and the fact that the value of $\lambda$ is directly connected with contribution of the corresponding POD mode, the eigenvalues and eigenvectors are sorted by the magnitude of the eigenvalues. Temporal coefficients $a_{i}$ and spatial eigenmodes $\phi_{i}$ are then computed using the following expressions:

$$
\begin{aligned}
& a_{i}=e_{i} \sqrt{N \lambda_{i}} \\
& \phi_{i}=V \frac{e_{i}}{\sqrt{N \lambda_{i}}},
\end{aligned}
$$

where $N$ is the number of samples and $V$ is the matrix consisting of all recorded samples transformed to the form of column vectors (matrix of size $M$ by $N$ size where $M$ is the number of pixels within one sample and $N$ is the number of the recorded samples). A dominant frequency of the selected mode $i$ can be then evaluated by the spectral analysis of the correspondent temporal coefficient $a_{i}$.
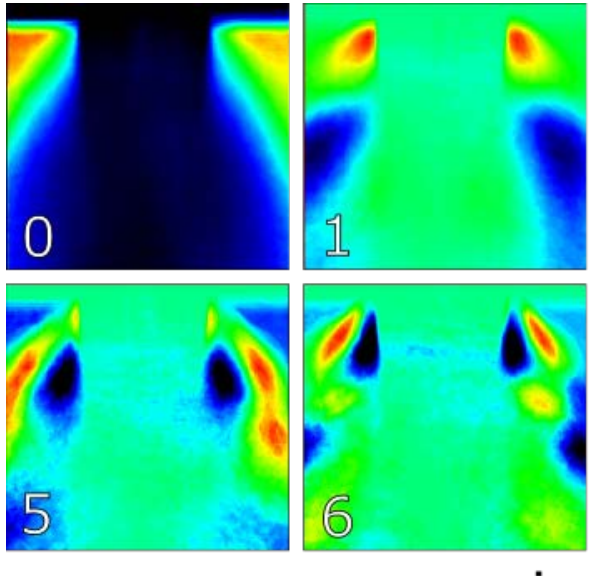

$\min$
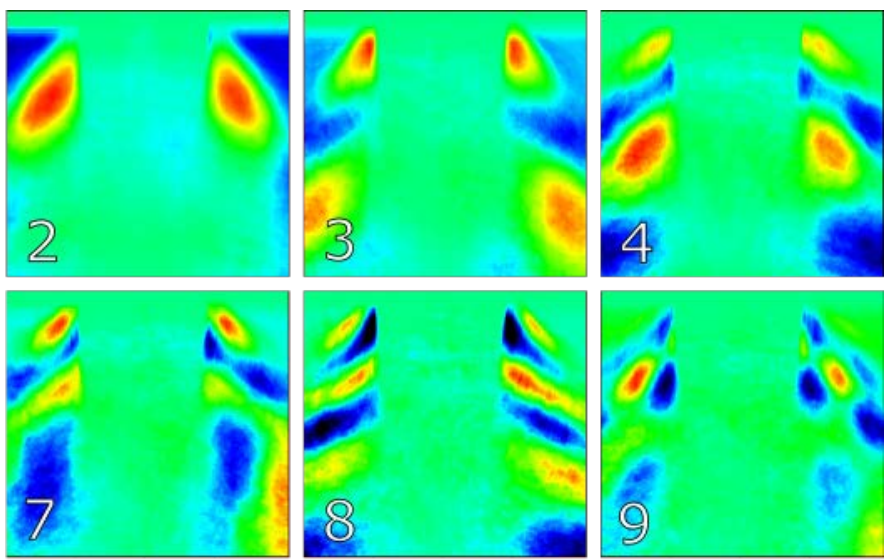

$\max$

Figure $6 P O D$ modes at $G L R=2.5 \%$. Numbers in images correspond to the mode number. 
The POD modes can be interpreted as a flow fluctuations. It represents characteristic shapes occurring in the examined phenomenon and it also gives the rate of a given mode. The POD modes point to a dynamic behaviour of deforming liquid. The zero POD mode, see Figure 6 , represents the static structure, whose shape corresponds to the structure of the mean image. The other modes show the dynamics structures occurring in the examined series. It should be noted that the lower is the number of the POD mode, the more significant is its contribution. It can be seen in Figure 6 where the $1^{\text {st }}$ and $2^{\text {nd }}$ dynamic POD modes depict massive structures, while the images of the higher modes show more fluctuating properties of the flow structures.

The contribution of each mode to the total record is shown in Figure 7 a). The absolute magnitude of eigenvalues are plotted in Figure $7 \mathrm{a}$ ). It can be understood as the magnitude of significance of an individual POD mode. It is clear that the first three modes are dominant. The mode significance then decreases with the growing number of POD mode. The cumulative distribution of mode significance at $G L R=2.5 \%$, Figure $7 \mathrm{~b}$ ), shows that only around ninety modes can be used to capture $80 \%$ of main characteristics. The regime $G L R=10 \%$ shows a similar trend but it needs slightly more modes to reach $80 \%$ value of significance. The GLR $=5 \%$ needs more POD modes to reach the same level of significance. This is probably due to the noise caused by the stochastic nature of this particular regime. Even from the optical point of view, the process was relatively more unsteady compared to the other examined regimes. A slow convergence of the cumulative distribution to unity reflects the chaotic stochastic nature of the primary breakup process. The information about the internal twophase flow might be useful and describes this behaviour in a greater detail but it is out of the scope of the present paper.

When investigating a series of modes, see Figure 6 , it can be observed that the first five modes have a relatively consistent structure which also corresponds well to the shapes present in the recordings of the liquid breakup, see Figure 6 . The modes from $5^{\text {th }}$ and higher are slightly more difficult to understand. Complicated structures in higher modes are probably caused by the multiple bursts in the image. Due to the difficulties with describing these modes and due to their low eigenvalue magnitudes, the analysis of POD modes is restricted to the first five modes. Moreover, the images of POD modes in Figure 6 and raw recordings in Figure 8 are symmetrical. Thus, for further analysis, only a right half of the images is used.

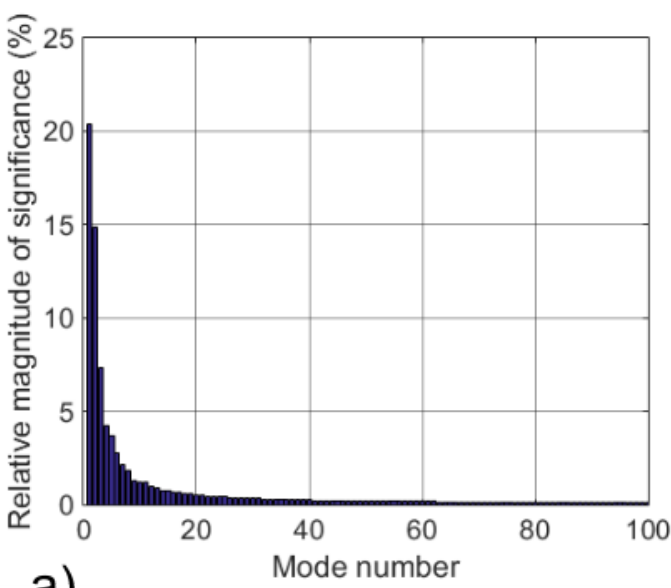

a)

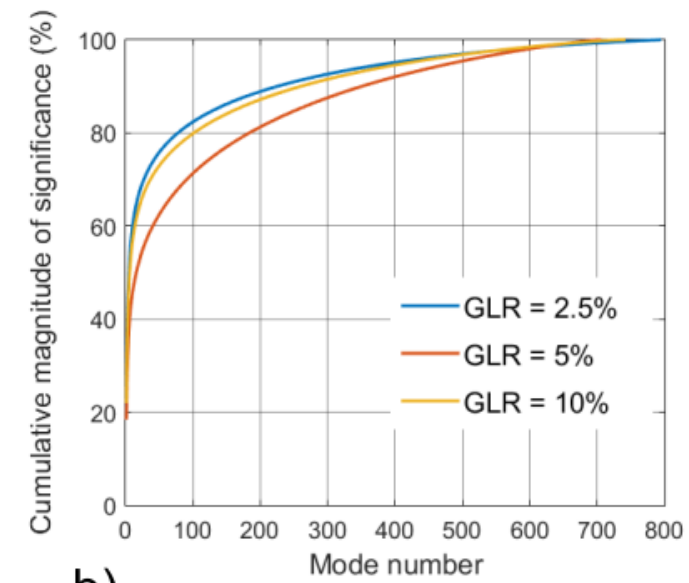

b)

Figure 7 Contribution of the POD modes. $G L R=2.5 \%$, similar distributions were observed also for other operating regimes: a) histogram of relative magnitude of significance of individual modes, b) cumulative magnitude of significance.

At the end of the POD analysis, the raw records were reconstructed from POD modes to verify the accuracy of the results. Fifty most significant POD modes were utilized in the reconstruction. In the 
case of $2.5 \%$ GLR, $76 \%$ of cumulative significance is contained within fifty most dominant POD modes. The values of the significance contained within the modes utilized for the reconstruction of regimes: $5 \%$ and $10 \%$ of GLR are $49 \%$ and $72 \%$ respectively.

A comparison of the original images and the corresponding reconstructions are shown in the following set of images. It is clearly depicted that the main gas-liquid structures are well captured, in spite of the fact that a relatively small amount of POD modes were utilized, see Figure 7 . It can be seen that the regime of $G L R=5 \%$ seems to be more blurred than other regimes, which corresponds to the results discussed in previous paragraphs.

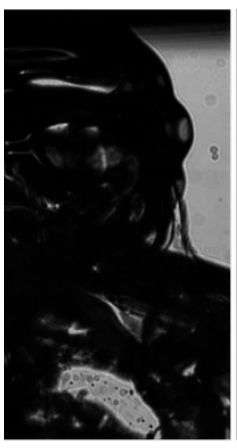

a)

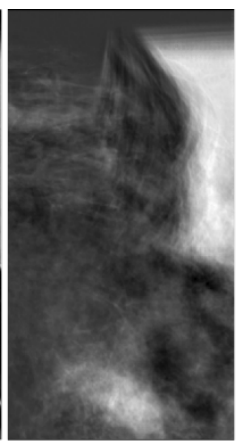

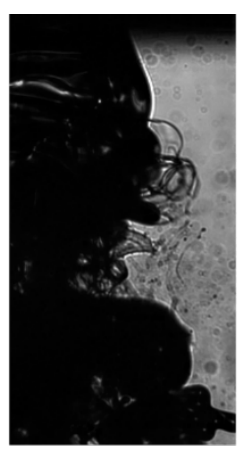

b)

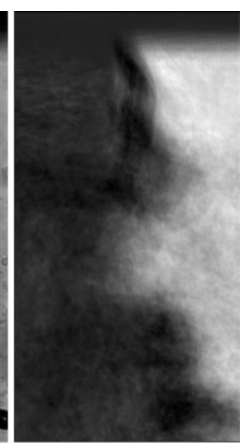

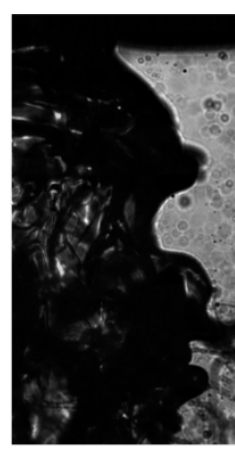

c)

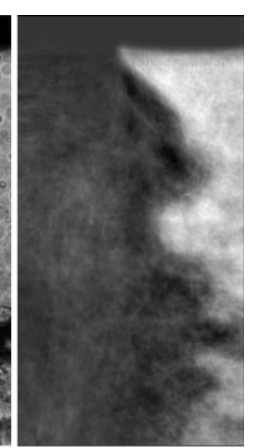

Figure 8 Comparison of raw images (selected snapshots) and POD reconstruction for all operating regimes: $a$ ) $G L R=2.5 \%, b$ ) $G L R=5 \%, c) G L R=10 \%$. The original raw image is on the left and the corresponding POD reconstruction is on the right.

\subsubsection{PDA data processing}

The PDA system measures the droplet velocity in two directions, axial and radial, and the droplet diameter. Thus, it enables to calculate basic flow characteristics such as mean and RMS velocities in both directions, mean diameter (D10), Sauter mean diameter (D32) and to compute variables such as a liquid flux.

The PDA system cannot obtain any information about the gas motion directly; it is necessary to use a simplification based on the Stokes number (Stk), common in spray applications, e.g. (Santolaya et al. 2010, Jedelsky and Jicha 2013), see equation 7 in the following section. It is assumed that the smallest particles, smaller than $5 \mu \mathrm{m}$ in diameter, follow the gas motion smoothly; they typically reach $S t k<1$, whereas larger droplets, $D \geq 20 \mu m$, typically reach Stk value 1 and higher. This is valid for majority of examined axial positions. In the positions close to the atomizer, 5 and $10 \mathrm{~mm}$, where droplets still might experience the secondary breakup, the Stk of small droplets does not follow the previous statement sufficiently i.e. they does not follow the gas motion. However, we use the same criterion because the information should still give an insight into the main features of the flow.

\subsubsection{Dimensionless numbers}

To describe the forces acting on the liquid during the deformation process and gas expansion, dimensionless numbers are used. The first number introduced here is the liquid Weber number (We) comparing the inertial forces with forces of surface tension, see equation 4 , where $U_{L}$ is the liquid or droplet velocity (measured by the PDA system), $D$ is a characteristic dimension, diameter of the liquid fraction. In the case of individual droplet, $D$ is related to its diameter whereas in the case of expanding liquid structure, it relates to the outer diameter of the structure.

$$
W e=\frac{\rho_{L} \cdot U_{L}^{2} \cdot D}{\sigma}
$$


A dimensionless number describing the ratio of viscous and surface tension forces is the Ohnesorge number (Oh), equation 5 .

$$
O h=\frac{\mu_{L}}{\sqrt{\rho_{L} \cdot D \cdot \sigma}}
$$

The Capillary number is used to determine the ratio of viscous to surface tension forces in a different way. Here the viscosity is linked with the liquid velocity as can be seen in the equation 6 .

$$
C a=\frac{\mu_{L} \cdot U_{L}}{\sigma}
$$

Stk of droplets was calculated using PDA data, according to the findings of (Santolaya et al. 2010, Jedelsky and Jicha 2013) who derived the following equation for a droplet flowing in the spray:

$$
S t k=\frac{\rho_{L} \cdot D^{2} \cdot\left|\left(U_{d}-U_{G}\right)\right|}{18 \cdot \mu_{G} \cdot Z}
$$

where $Z$ is a decay constant, $U$ is velocity, and $\mu_{G}$ is gas dynamics viscosity. Subscript $d$ is related to the measured droplets.

\section{Results and Discussion}

This section describes a liquid deformation on the basis of back-illumination records and PDA data. The characteristic events of liquid breakup are distinguished using the POD and frequency analysis. Combining the information from the raw recordings and the data presented in this work, an idealised image sequence of breakup mechanism is achieved to describe the liquid breakup process. Further, an analysis of the simplified breakup mechanism is carried out using dimensionless numbers.

\subsection{Frequency analysis}

The results show a frequency analysis obtained from the selected points in the RMS images, Figure 4. These results are compared with the characteristic frequencies of POD modes in Table 3. The measured frequency obtained from the raw recordings corresponds relatively well with the dominant frequencies of the majority of the first five POD modes. Especially the most significant modes have a similar dominant frequency. The only exception is the regime of $G L R=5 \%$ where the first POD mode frequency demonstrably differs and the following frequencies show a higher deviation than other GLR regimes. This can be explained by plotting the frequency spectrum (Figure 9) of the given POD mode. The dominant frequency is truly in 4.78 but there are more distinguishable peaks, one of them in $12.58 \mathrm{kHz}$, which corresponds to the frequencies of higher modes. The noise in the frequency spectrum of this regime is attributed to its stochastic nature as discussed earlier in the section 2.4.3. Similarly, a deviation of dominant frequencies for the raw record and POD modes is probably influenced by the same mechanism. 
Table 3. Dominant frequencies of the raw recordings and POD modes.

\begin{tabular}{|c|c|c|c|c|c|c|}
\hline \multirow{2}{*}{ GLR } & \multicolumn{7}{|c|}{ Dominant frequency } \\
\cline { 2 - 7 } & \multirow{2}{*}{$\begin{array}{c}\text { Raw } \\
\text { record }\end{array}$} & 1 & 2 & 3 & 4 & 5 \\
\cline { 2 - 7 }$\%$ & \multicolumn{7}{|c|}{$\mathrm{kHz}$} & 13.20 & 4.03 \\
\hline 2.5 & 12.61 & 12.58 & 12.58 & 12.58 & 13.21 & 4.65 \\
\hline 5 & 12.34 & 4.78 & 13.21 & 13.21 & 9.36 & 6.55 \\
\hline 10 & 13.33 & 13.37 & 13.37 & 12.57 & & \\
\hline
\end{tabular}

Frequency spectrums of higher POD modes, typically the fifth mode and higher, show a similar behaviour. The noise tends to increase; detecting of dominant peaks is thus difficult.

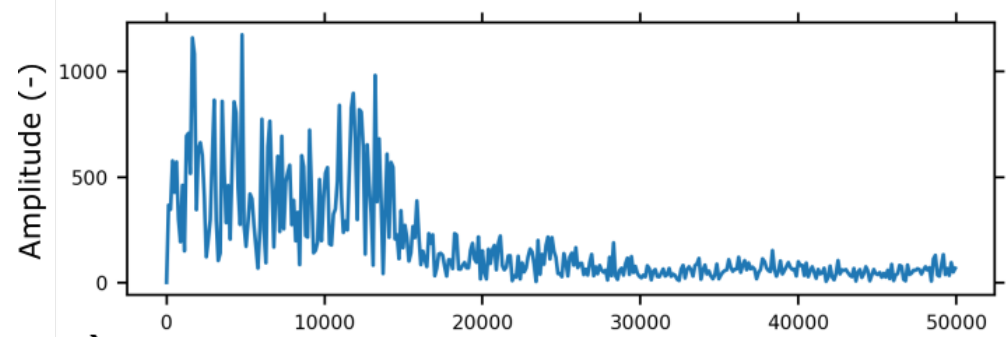

a)

$\mathrm{f}(\mathrm{Hz})$

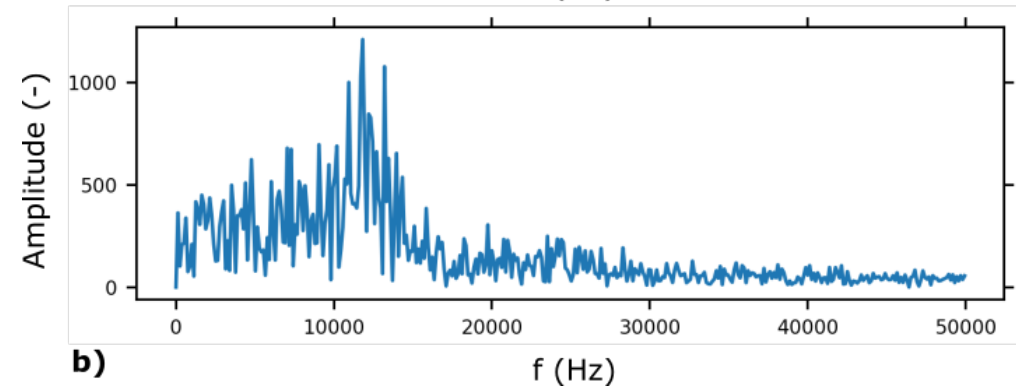

Figure 9 Regime $G L R=5 \%$, frequency spectrums of the POD modes: a) first mode, $b$ ) second mode.

From the above-mentioned description, it can be stated that the shapes occurring during the liquid deformation correspond mainly to the first four POD modes. The rest of the modes is more difficult to describe using a frequency analysis. However, the fifth mode is used to support a discussion over the breakup mechanism in the following section.

\subsection{POD modes}

The physical meaning of the POD modes can be described as flow fluctuations. The exception is zero POD mode which represents the static structures in the examined images series. In other words, it shows mean values of the pixel intensity in the image series. Figure 10 shows POD modes together with a mean image (zero mode), the first from the left side. With the increase of GLR, the mixture, tends to narrow mainly at the bottom of the image, as indicated by a dark blue colour. It means that the liquid is dispersed over a wider area. This can be attributed to a higher amount of gas which is supposed to result in generation of larger gas bubbles that force the liquid to disrupt earlier. Moreover, at higher GLR, the spray is less dense due to a lower fuel mass flow which also influences the light intensity in the images. 
The following modes (1-5) point out the dynamics shapes occurring during the liquid deformation. In general, the areas with the highest and the lowest pixel intensity, red and blue colour respectively, show the regions of a high dynamic importance. When investigating a series of modes, several features can be observed. For all regimes, the first mode is characterized by a relatively simple structure which is similar to what can be seen in raw images. A transition between the colours (green-blue-red) creates the structure similar to the shape of the expanding liquid. It can be compared to the cone shape which expands in radial direction and flows downstream. As GLR grows, the cone shape tends to move closer to the atomizer. This is a manifestation of the increase of gas mass flow which changes the momentum ratio between the gas and liquid. In other words, the liquid is deformed earlier because the gas bubbles are larger and the amount of accelerated liquid mass is comparably smaller.

Mode characteristics show that during the liquid deformation process the gas expansion is clearly distinguishable and the liquid diameter enlarges from the initial diameter to the edges of the examined area. Then it propagates downstream until it breaks up. This idealised description of the breakup mechanism was used for filtering of raw recordings according to the shapes occurring in the first five POD modes. Raw recordings, which enabled point tracking, were examined using the liquid tracking method, see Figure 11 in the next section. The liquid tracking method was performed manually for selected events which showed the clear and distinguishable development of the deformation. Each operating regime was examined by 10 different events. The resulting curves, shown in Figure 11, were obtained by averaging the data from the measurements. 

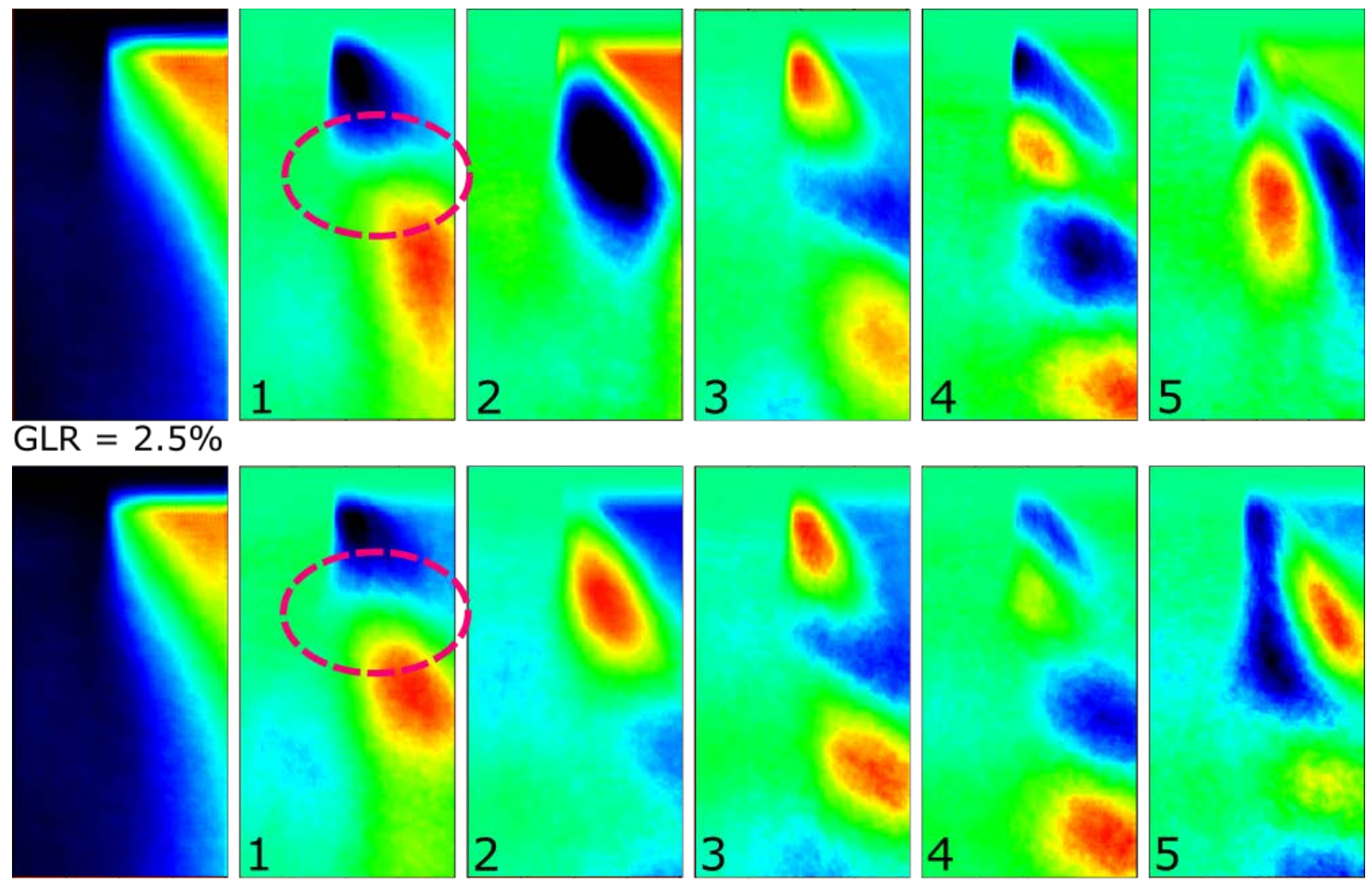

$$
\mathrm{GLR}=5 \%
$$
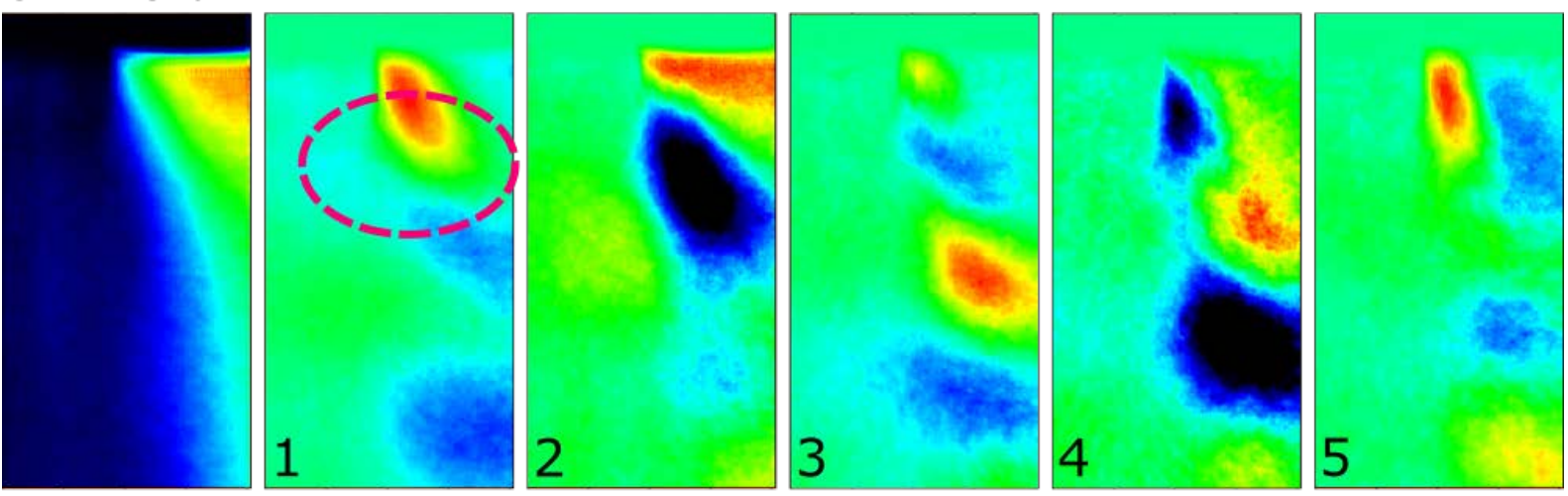

$\mathrm{GLR}=10 \%$

\section{$\min$}

Figure 10 First five POD modes (0-4) for all three examined operating regimes. The pink circles highlight the tendency of the bubble initial deformation occurs closer to the atomizer when GLR is increased.

\subsection{Liquid deformation mechanism}

The images from the back-illumination technique were obtained in the near-nozzle area - the area of liquid initial deformation. The following description is based on the visual evaluation of the recordings which were filtered to the events occurring in the first five POD modes. Further, the liquid tracking method was applied to estimate the velocity and dimensions of the liquid fractions during deformation.

\subsubsection{Visual observation and liquid tracking method}

Two different image resolutions were used for visual evaluation of breakup mechanism, see Figure 11 and 12. The recordings show that the emerging two-phase mixture consists of liquid bulk and small gas bubbles inside. When GLR is low (2.5\%), bubble expansions are easily detectable and individual bursts can be observed, see Figure 11. During the bubble expansion, the liquid extends to a certain radial distance which tends to be dependent on the gas bubble size. Then it flows mainly in 
the axial direction. It means that the liquid radial extension takes place only when the gas is surrounded by the liquid. When the liquid disrupts, usually from below, the gas expands mainly in the axial downstream direction because this flow is not blocked by the consistent liquid on the periphery. From visual observation of Figure 11 and 12, and the above description, it can be deducted that the breakup mechanism is based on the superposition of the two main events:

The mechanism a) is related to the radial extension of the liquid until the point where the liquid is disrupted. The expanding gas forms the liquid into the shape of annular liquid sheet, bubble, which eventually breaks up into individual fractions, ligaments and droplets. These structures flow with the velocity given by the initial gas expansion. The further breakup of ligaments is caused by the instabilities generated during the breakup which leads to a vibration of the ligaments and droplets. This mechanism is amplified by the surface tension forces which are responsible for the breakup of ligaments as pointed out in (Mlkvik et al. 2015). This was especially visible in the recordings in Figure 12.

The mechanism b) takes place in the spray core, see stage 6 in Figure 12. After the initial liquid deformation and initial breakup, the liquid fractions are rapidly accelerated in the axial direction. Assuming that this process occurs periodically, the accelerated two-phase mixture interacts with the liquid structures and droplets generated by the previous burst. This mechanism enhances the breakup process and is responsible for secondary breakup in the core of the spray which generates small droplets.
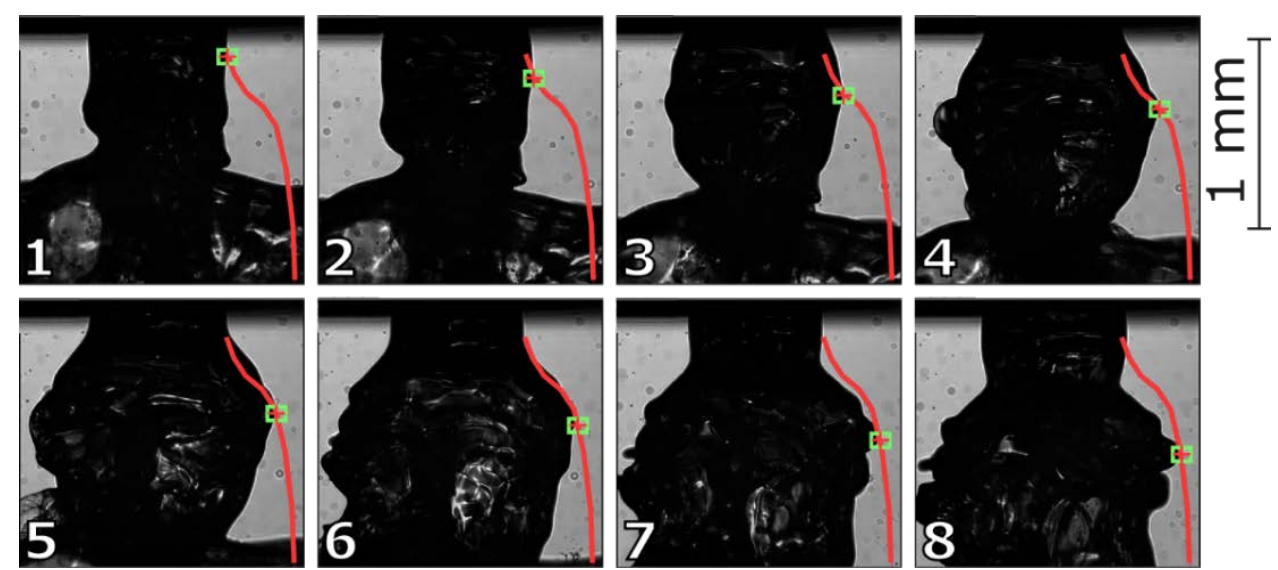

Figure 11 Image sequence capturing the liquid deformation caused by the gas bubble expansion. GLR $=2.5 \%$, frame rate $100,000 \mathrm{fps}$. The images also include liquid tracking shown by a red line and a green square. The liquid deformation was identified by a disruption of the liquid column emerging from the orifice, stage 1 in the Figure. This disruption then develops due to the gas expansion. The liquid tracking method then followed the characteristic shape until the bubble broke up. The tracking was done manually on selected images series.

The size of gas bubbles vary with time, which leads to different dimensions of extended liquid structure. If the gas flow rate increases, the bursting frequency grows, which confirms the findings of (Gadgil and Raghunandan 2011). A higher amount of gas causes the bubbles to vary in size even more. When the gas bubble is relatively small, it might remain trapped inside the liquid and continues as a frozen liquid-gas structure. It consequently breaks up due to the interaction with accelerating bubble flowing behind. Thus, the liquid breakup should not be limited to just one mechanism - the bubble expansion. From the observations, it is obvious that the breakup process proceeds as a superposition of several mechanisms where the two dominant mechanisms are described above.

The mechanism of liquid deformation seems to be the same for all three operating regimes. However, the liquid deformation process differs from the previously published results on 
effervescent atomization. The main difference is that, in our case, no solid liquid column which divides two following bubbles was observed, as noted by (Gadgil and Raghunandan 2011). Comparing to their experiments the initial deformation in our case starts immediately behind the orifice. The (Gadgil and Raghunandan 2011) observed undisturbed liquid column in the near-nozzle area which breaks up in the bursting distance.
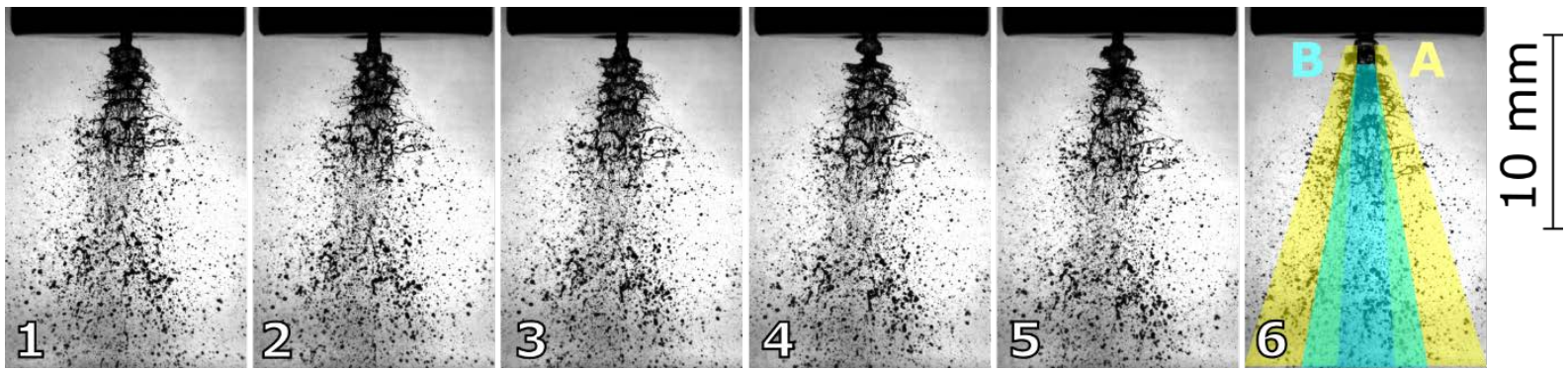

Figure 12 Image sequence showing the liquid breakup and spray creation. GLR =2.5, camera's frame rate 30,000 fps.

\subsubsection{Influence of GLR on the liquid breakup}

When comparing the operating regimes, they differ in the so-called bursting frequency as noted in the section 3.1, previously commented by (Gadgil and Raghunandan 2011). A higher amount of atomizing gas causes the discharging liquid structures to extend in radial direction even further and to break up closer to the atomizer. The extension rate can be evaluated using RMS images, Figure 13, which shows a standard deviation of the pixel intensity in the examined image sequence (Marchi et al. 2010). The regime $G L R=2.5 \%$ is characterized by the high intensity areas close to the exit orifice. This shows that in these areas the liquid experiences a detectable and rapid extension. When the GLR increases, the intensity of previously mentioned areas reduces. This causes the bursts to occur more frequently and with various bubble sizes, which consequently leads to a worse detectability of the individual bursts. Moreover, the spray core, represented by dark blue colour, extends with the increase of GLR. This can be visible in RMS but also in the zero POD modes.

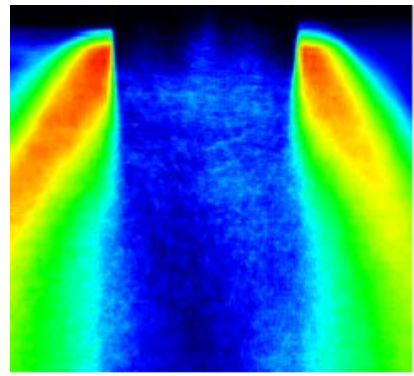

a)

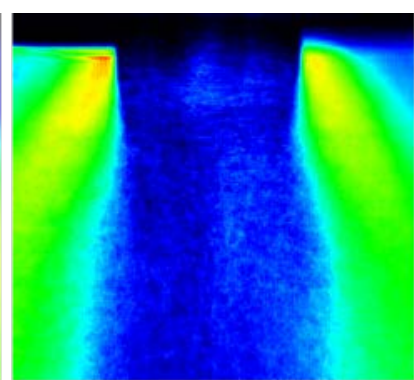

b)

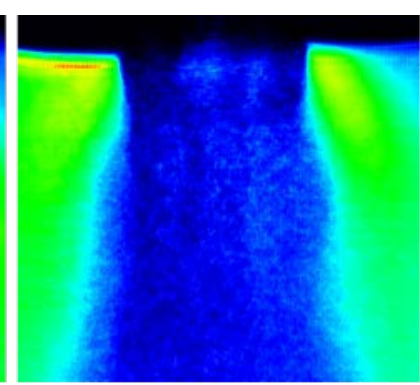

c)

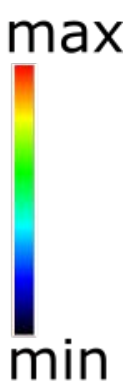

$\min$

Figure 13 RMS images for operating regimes: a) $G L R=2.5 \%, b) G L R=5 \%, c) G L R=10 \%$.

\subsection{Physical description of the liquid breakup}

Based on the visual observation, the frequency and POD analysis enables us to derive a simplified model of liquid breakup mechanism. Several assumptions have to be made to describe the physical background of the breakup. Firstly, this description is limited to the deformation which is most common, i.e. it corresponds to the shapes of the first five POD modes. Secondly, with estimation of the evolution process of inner gas pressure, we assume an ideal adiabatic expansion. Thirdly, the 
characteristic length, i.e. diameter used in dimensionless numbers, is taken by the tracking method from the outer diameter of the expanding liquid, see Figure 11.

\subsubsection{Description of the breakup mechanism}

A simplified schematic drawing of liquid breakup, based on the effervescent atomization, is shown in Figure 14 and described step by step below. It is assumed that the gas expansion and following breakup can be described by the first five states in Figure 14 - the expansion of the gas bubble no. 1 . To explain the superposition of the two following bursts, states 5-7 are used.

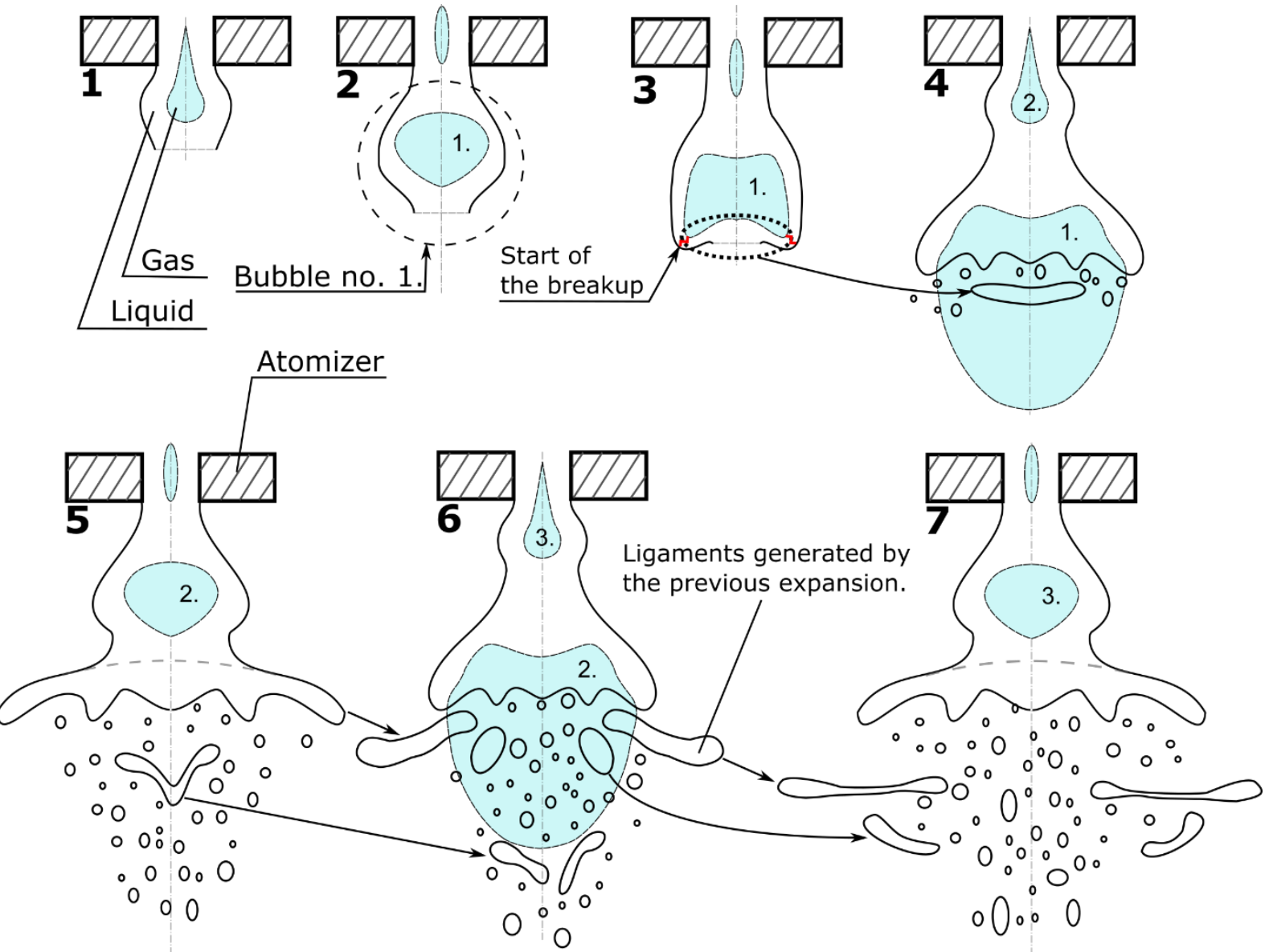

Figure 14 Simplified drawing of the liquid deformation and primary breakup deducted from the high-speed backillumination recordings. The breakup process was derived from two different image resolutions i.e. figures 11 and 12 . Figure

11 was used for detailed examination of the liquid initial deformation whereas figure 12 was used to examine the

interaction of the two following bubble bursts.: 1) Discharge of bubble. 2) Bubble expansion. 3) The radial expansion is nearly finished. 4) Start of the liquid breakup. 5) Radial deformation of the liquid due to the gas expansion is finished and the axial acceleration enhances the breakup in the core of the spray. 6) Large droplets and ligaments, created by the first gas bubble expansion, are now accelerated by the following expanding gas bubble. 7) Ligaments are elongated, accelerated, and consequently disrupted by the combination of the acceleration of the expanding gas and by the interaction with the ambient atmosphere.

1) The process starts by discharge of the gas bubble after passing through the atomizer's exit orifice. The gas is squeezed during the passage. When it is discharged, it is partly decelerated by the liquid which flows in front of the gas. Moreover, after leaving the orifice, the gas, expands in a radial direction as shown in the Figure above.

2) At this point, the gas bubble has completely left the exit orifice so it can freely expand in all directions. However, a dominant direction of the liquid movement is axially downstream. It means that the gas, trapped inside the liquid, is also affected by inertial forces of the 
surrounding liquid. On the front side, taken from the flow direction, the gas slows down by acting on the liquid stream at the front. On the rear side, the liquid stream, flowing behind the gas, pushes on the gas from above. This eventually leads to the shape similar to that of the stage 3:

3) This shape is modelled by the gas expansion in radial direction, which is almost finished, and by the liquid streams in front of and behind the gas bubble. The gas tends to wrap the liquid stream at the front. This movement makes the liquid sheet thinner creating spots where the breakup starts as it is highlighted by the red lines in the figure above.

4) The liquid bulk right after the initial breakup is shown in this state, disrupted from below, as predicted in the previous state. A radial extension of the liquid then continues due to the inertial forces. Gas expands mainly in the axial downstream direction because it is not surrounded by the liquid completely. Usually, in the centre of the spray, large liquid structures, generated by the liquid breakup, are present as shown in the figure above. The following gas bubble is discharged from the orifice and the next bubble explosion is initiated.

5) In this state, the gas should be almost completely expanded, and the deformation of the rest of the liquid structure is a manifestation of inertial forces. The liquid fractions, present in the centre, are rapidly accelerated by the expanding air, which causes their deformation i.e. thinning. The edges of the liquid core are also thinned. Moreover, this process tends to generate unstable vibrating structures. This unstable nature enhances their breakup into smaller fractions and individual droplets; a secondary breakup, as shown in the stage 6 .

6) This stage shows the two breakup mechanisms described earlier in 3.3.1: a) The edges of the liquid core break up into ligaments and large droplets due to the instabilities on the liquid surface generated during the deformation process. The liquid fractions on the edge generated by the previous burst flow downstream and elongate in the radial direction. This is a manifestation of drag force from the expanding gas bubble and the ambient atmosphere combined with the surface tension and inertia forces. This process continues to the stage 7.

b) The structures close to the spray main axis are accelerated by the expanded gas, which eventually causes their thinning and consequently breakup.

7) The ligaments and other liquid structures flow axially and are elongated due to the forces acting on them. The gas expansion has accelerated the liquid structures mainly in the core of the spray which produces relatively small droplets. The expansion of the previous gas bubble should be completed, and liquid fractions are accelerated mainly axially. As the distance from the atomizer increases, the gas is slowed down due to the interaction with the ambient atmosphere.

\subsubsection{Analysis of the forces acting on the liquid}

To describe the ratios of forces acting on the liquid during the gas expansion, dimensionless numbers are used. The following description is limited to the states when the gas is trapped inside the liquid i.e. states 1-4 in Figure 14. From the measured data, it was deducted that the gas bubble expansion starts at an initial diameter, roughly the same as the exit orifice, and continues to approximately double diameter, where the initial disruption occurs.

Velocity profiles are shown in Figure 15 to explain the spatial evolution of the liquid deformation. Data are taken from the liquid tracking method mentioned earlier. It was revealed that for these fitted velocity curves the $\mathrm{R}$ square (coefficient of determination) reaches the values of 0.6 for $G L R=2.5 \%$ which fit relatively well comparing other regimes. However, in the cases of $5 \%$ and $10 \%$ of GLR, the $\mathrm{R}$ values are 0.24 and 0.05 respectively, which points out an inconsistent data distribution. It is caused by the increased velocity of deformation which enhances distortion of data obtained by the liquid tracking method. Thus, for further analysis of the liquid deformation, we 
assume just the regime of $G L R=2.5 \%$ while other regimes are discussed just to point out the trends related to the examined phenomenon.

Both velocities, radial and axial, have similar profiles assumed as quadratic. The liquid accelerates at the beginning from the initial discharging velocity to the maximum (states 1 and 2 in Figure 14). Further, the velocities decrease, which is a manifestation of the stage when the gas has expanded to its momentary maximum (stage 3 ) and the liquid deformation tends to slow down until it reaches the point of the first disruption of the liquid (stage 4). It shows that the deformation does not have a constant velocity but depends mainly on the gas state, i.e. gas inner pressure.

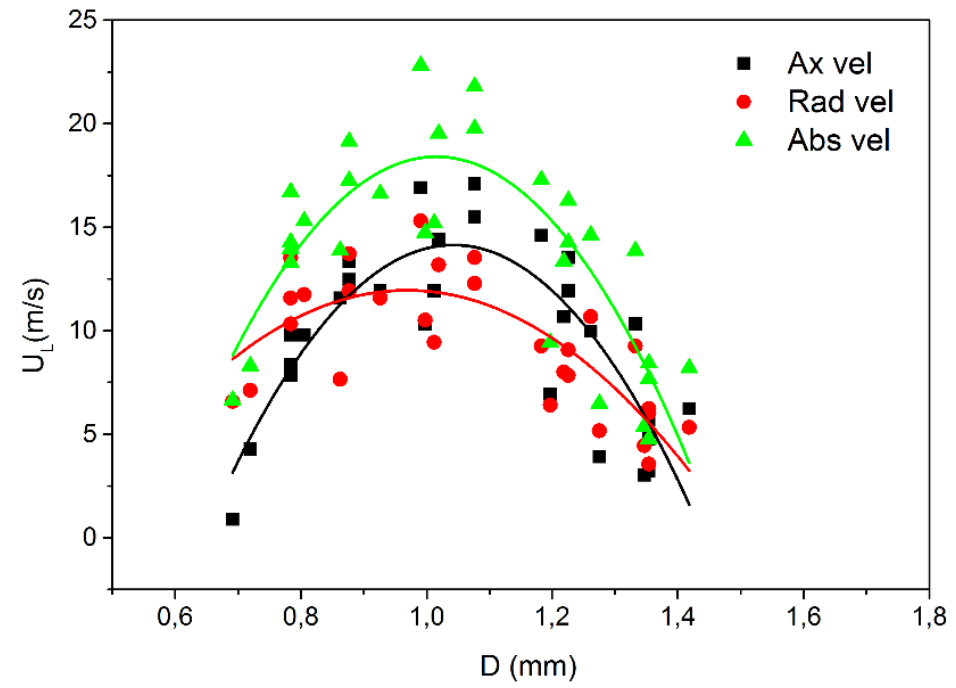

Figure 15 The liquid velocity distribution obtained from liquid tracking method for the GLR $=2.5 \%$.

The Capillary number (Ca), equation 6, represents a relative effect of viscous forces versus surface tension, see section 2.4.5. The $\mathrm{Ca}$ is influenced by the liquid velocity which changes with a liquid diameter. When the liquid accelerates (starting at $\mathrm{Ca} \approx 4$ ), the importance of viscous forces related to the velocity also grows. When the radial expansion reaches the maximum, which corresponds to the $C a \approx 14$, the liquid flows mainly downstream and the absolute velocity decreases to the stage where $\mathrm{Ca} \approx 3$. It shows that the viscous forces should influence the speed of deformation and consequently the maximal radial extension of liquid. In our case, the Capillary number is relatively high; therefore, the surface tension forces should have only a minor effect on the liquid deformation process. Thus, the viscosity should be a more important parameter influencing the liquid motion. Different situations occur after the liquid has experienced the primary breakup. Then the surface tension forces should play a dominant role in the breakup process as noted in (Mlkvik et al. 2015; Faeth, Hsiang, and $\mathrm{Wu}, 1995)$. 


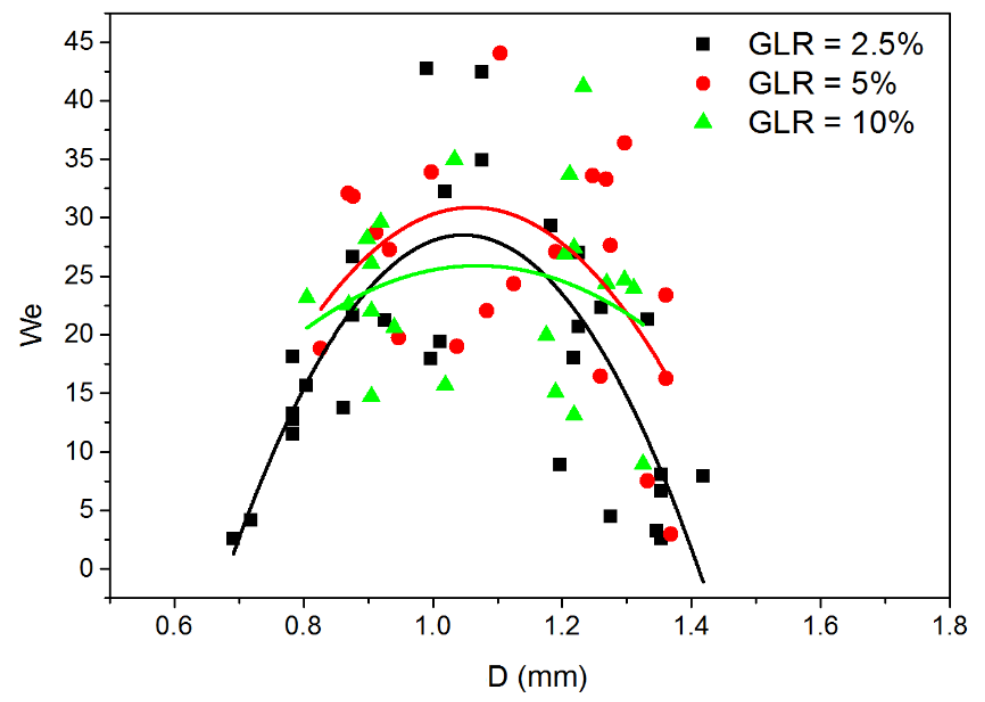

Figure 16 Weber numbers depending on the liquid outer diameter.

The Weber number (We), equation 4, expresses the ratio between the liquid inertia and the surface tension of the fluids outer interface 2.4.5. It should be noted that the obtained values of Weber number were calculated from the liquid tracking method where only an outer liquid diameter and its velocity were measured.

Figure 16 shows a dependence of We on the diameter of liquid body during the gas expansion for all examined regimes. It can be seen, that the fitted curves describe well the regime of $G L R=2.5 \%$ but very poorly the rest of the regimes as mentioned earlier in this section. Similar to the velocity, the Weber number grows, according to concave quadratic function curve, until it reaches a local maximum and then it falls down. The Weber number depends on the square of velocity; thus, it is more sensitive to the velocity than to the liquid diameter.

The Ohnesorge number (Oh), equation 5 , gives in relation viscosity and surface tension. The calculated $\mathrm{Oh}$ is in the range from 3 to $4.5 \times 10^{-3}$. The results of (Faeth, Hsiang, and Wu 1995) and (Mlkvik et al. 2015) show that using We and Oh numbers the stage of the liquid breakup process can be determined. The threshold between deformation and breakup regime is when $W e_{\text {crit }}=10$ and it weakly depends on the Oh value. The present study differs due to the fact that liquid is surrounded from the inside and outside by the gas which means that $W e_{c r i t}$ is not a relevant parameter for our study. The evolution of We during the gas expansion, Figure 17, shows that the breakup does not occur at the maximal value but it is a consequence of the motion right after the deformation is finished. Thus, the drag forces from ambient atmosphere do not significantly contribute to the primary breakup.

The curves describing We show that it reaches higher values in the case of $G L R=5 \%$, and lower values for $10 \%$. This can be attributed to the fact that the increase of gas amount causes that the initial disruption occurs sooner, transition from stage 3 to 4 in Figure 14, and it also propagates faster. Then the liquid tracking method reaches its limit of temporal resolution, a camera frame rate at a given resolution. 


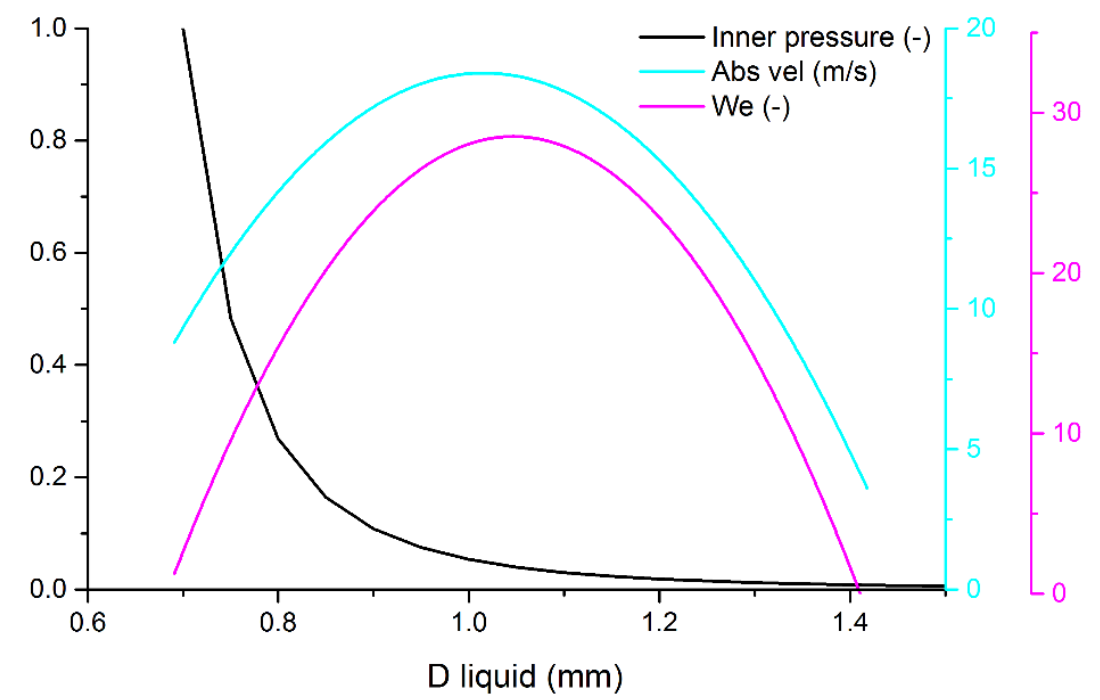

Figure 17 Spatial development of the pressure inside the gas bubble, liquid absolute velocity and Weber number. Operating regime $G L R=2.5 \%, 10$ bubble bursts were analysed.

When assuming the ideal gas and adiabatic expansion, the evolution of the inner gas pressure should be driven mainly by the change of the gas volume, i.e. by the liquid diameter when assuming that the gas forms an ideal sphere. The pressure, discussed here, is the overpressure between the inner gas and the ambient atmosphere and it is plotted by the relative scale (from 0 to 1 ), see Figure 17. The pressure starts at its maximum and goes exponentially down to zero with a liquid diameter according to the equation of state. The main pressure drop occurs right at the beginning of the expansion, which also corresponds to the raw recordings. The pressure evolution supports also the velocity and Weber number profiles where the liquid accelerates rapidly at the beginning of deformation.

Liquid acceleration is caused by the gas expansion. However, the initial liquid disruption is a consequence of superposition of more factors. When the pressure drops to zero, velocity and We start to decrease as well. A decreasing character of velocity can be described on the basis of liquid deformation and its states shown in Figure 14. When the liquid bubble reaches the maximum diameter (stage 3), deformation continues mainly in the axial direction, which causes thinning of the liquid from below. Contraction of the liquid enhances deformation and breakup. The effect of viscous forces decreases, which enhances the gas propagation through the liquid phase. This process consequently leads to the generation of cracks in the liquid which then propagates further due to the surface tensions forces. A similar process occurs during the planar liquid sheet atomization (Lefebvre, 1988).

\subsection{Spray spatial evolution}

Five axial positions were examined by the PDA system for all operating regimes. In each axial position, one radial profile was measured. The results are plotted in the figures below. In internallymixed twin-fluid atomizers, the spray is created by the interaction of liquid and expanding gas. The gas enhances deformation and consequently the liquid breakup, accelerating thus the liquid, as discussed in the previous section. In the early stage of the gas expansion, the liquid deforms in radial and axial direction. However, the resulting dominant motion is in axial direction.

Velocity vectors showing the gas and liquid velocity indicate how the influence of GLR. The velocity in the exact centre of the spray should be strictly axial due to a symmetric construction of the atomizer (Sovani, Sojka, and Lefebvre 2001) and a statistically symmetric deformation process, see 2.4.3. In present results, the velocity in the centre has a small radial component which was probably caused by the inaccurate alignment of the atomizer to the coordinate system of the PDA 
system. Gas velocity is demonstrably higher in the centre of the spray whereas the liquid has higher axial and radial velocities outside the spray core. This feature decreases with axial distance, which is a manifestation of interaction of droplets with flowing gas and ambient atmosphere; this enhances mixing processes inside the spray. However, as noted by (Lasheras, Villermaux, and Hopfinger 1998) and (Jedelsky and Jicha 2014), the mechanism described as the overshooting phenomenon should also occur. Small particles accelerate in the near-nozzle region, in our case in the axial distance less than $10 \mathrm{~mm}$, and then they slow down due to drag forces and the interaction with the ambient atmosphere. In contrast, the large particles, which contain the majority of liquid, require a longer time and distance to accelerate to the speed of flowing gas. Afterwards, their velocity is kept relatively constant due to high droplet inertia.

A radial component of velocity increases with radial distance from the centre of the spray. Moreover, a liquid phase seems to have much higher radial velocity than the gas, especially in the near-nozzle area. This correlates well with the previous description of the liquid breakup mechanism where the radial expansion generates large liquid structures and droplets. The axial velocity of the gas is given by discharge velocity and expansion right after the disruption of a liquid bubble. It confirms that in the near-nozzle region, two mechanisms are responsible for the spray generation. However, with a growing axial distance, the interaction between the ambient atmosphere and a discharging gas-liquid mixture grows. Therefore, the influence of the two above described breakup mechanisms vanishes with the axial distance.

When comparing the results from different operating regimes, it is evident that the increase of GLR takes effect by increasing the axial velocity mainly in the spray centre. However, the radial component remains relatively unaffected by the GLR. It indicates that a higher gas mass flow causes an earlier disruption of liquid. The expansion propagates mainly downstream after the liquid initial breakup. Thus, the increase of gas mass flow increases mainly the velocity in the spray core, which enhances the liquid breakup among the spray axis. This mechanism affects the droplet size distribution mainly in the spray centre as can be seen in the graphs below.

The fluctuating component of the velocity, RMS, shows that the highest variations in velocity are in the centre of the spray. The individual bursts are responsible for the unsteadiness in the spray (Jedelsky et al., 2008). The rate of fluctuations decreases with the axial distance due to deceleration of droplets by the interaction with the ambient atmosphere together with the superposition of the two following bursts, as noted in 3.4.1. The interaction of bursts can be seen for all operating regimes. An interesting fact was found for RMS velocity component when comparing operating regimes. With growing GLR, the RMS value tends to decrease for both the liquid and gas phase. The higher gas mass flow causes the bubble burst to occur more frequently, which generates more homogenous mixture. In other words, the higher frequency of bursts causes their stronger interaction, which improves the gas-liquid mixing.

Droplet size distributions, D10 and D32 profiles, show that in the centre of the spray, the mean droplet size is relatively small comparing the outer spray regions. The exception are locations close to the atomizer where the maximal droplet size is close to the centre. However, with the axial distance, the droplet size decreases in the core of the spray and increase at the edges. At the regime of $G L R=$ $2.5 \%$, the largest droplets, expressed by D32, are present in the centre of the spray close to the atomizer. This peak moves radially with axial distance, which leads to the peak at the edge of the spray, axial distance of $80 \mathrm{~mm}$. It refers to a radial migration of droplets outside the spray core. This is supported by the radial component of velocity and points out the importance of mixing gas and liquid phase within the spray. Thus, the peak of mass flow occurs firstly close to the centre and then it moves out and finally vanishes with axial distance. It shows that to achieve a well-mixed spray a certain distance is required, $40 \mathrm{~mm}$ in the examined case. 
When inspecting the mass flow profiles, expressed in percent of total mass liquid flow, it can be seen that the majority of the liquid mass flows in a certain distance from the centre, which is partly in contradiction with of (Jedelsky and Jicha 2012) who showed that majority of liquid mass is in the centre of the spray. This peak then moves out from the spray core and the result is a relatively flat profile at the distance $80 \mathrm{~mm}$ from the atomizer, which confirms statements discussed in the previous paragraph. Moreover, from the evolution of liquid mass flow profiles, we can estimate which breakup process is responsible for disintegration of majority of the liquid mass flow. There are two breakup mechanisms: the breakup in the spray core and the breakup at the edges, which are driven by different forces as discussed in sections 3.4.1 and 3.4.2. When investigating the near-nozzle area, the mass flow profiles show that the majority of liquid does not flow in the spray core. The peak in the profile refers to the importance of superposition of the breakup mechanisms. It means that the liquid deformation, due to the gas expansion, is needed to increase the Weber number by radial extension, which enhances the liquid disruption. Further, the gas expansion takes place within the spray centre where it accelerates the two-phase mixture. In addition, the influence of drag forces is important at the edges of the spray where large droplets and ligaments appear; this causes mixing. This process leads to the equalization of the liquid mass flow as it is clearly seen in the mass flow profiles. Thus, the combination of the breakup processes is responsible for the disintegration of majority of the liquid mass. This applies to all examined regimes.

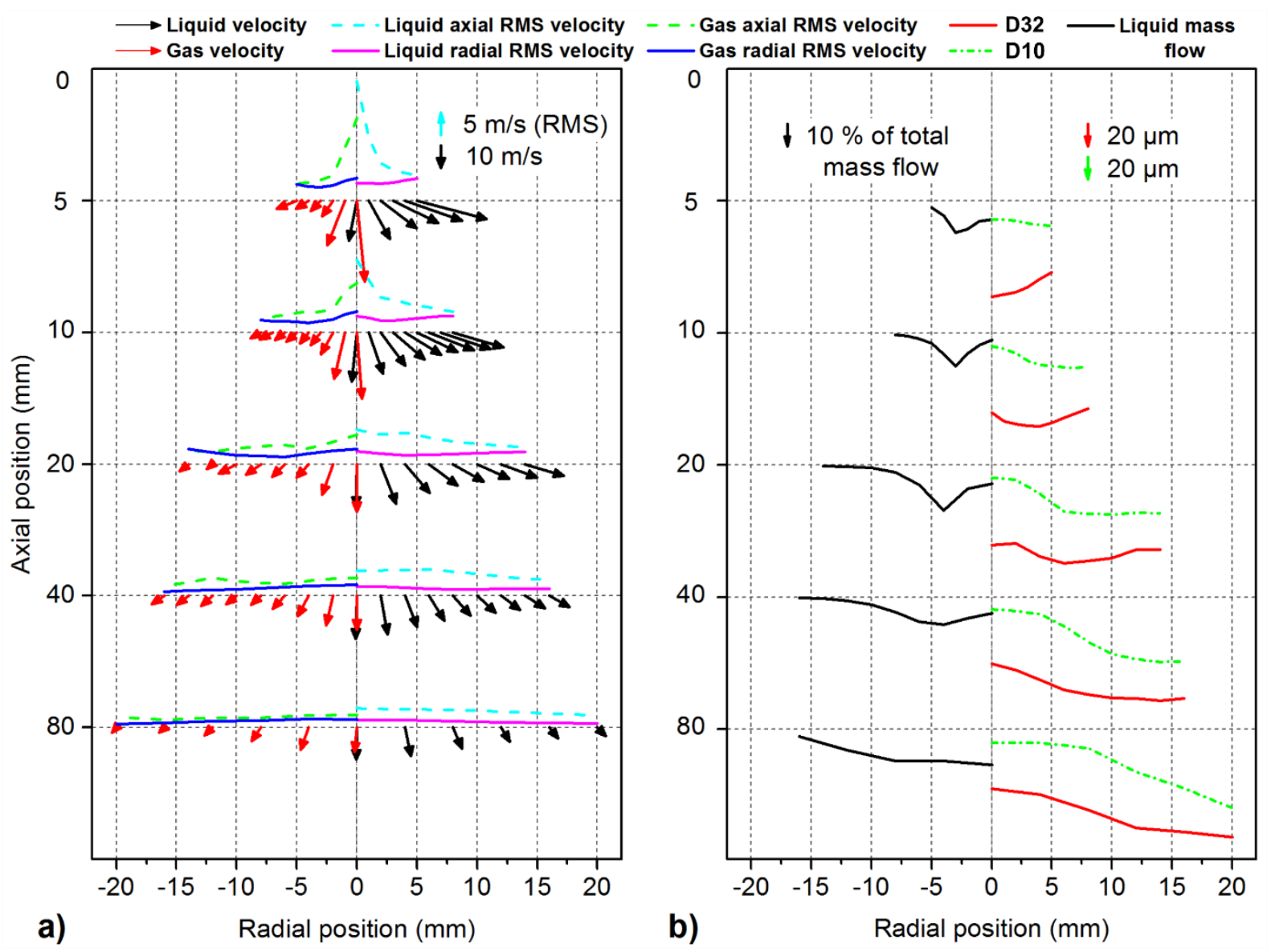

Figure $18 G L R=2.5 \%$, a) mean velocity vectors with RMS velocity profiles for both gas and liquid phase, b) liquid mass flow, D10 and D32 profiles. 

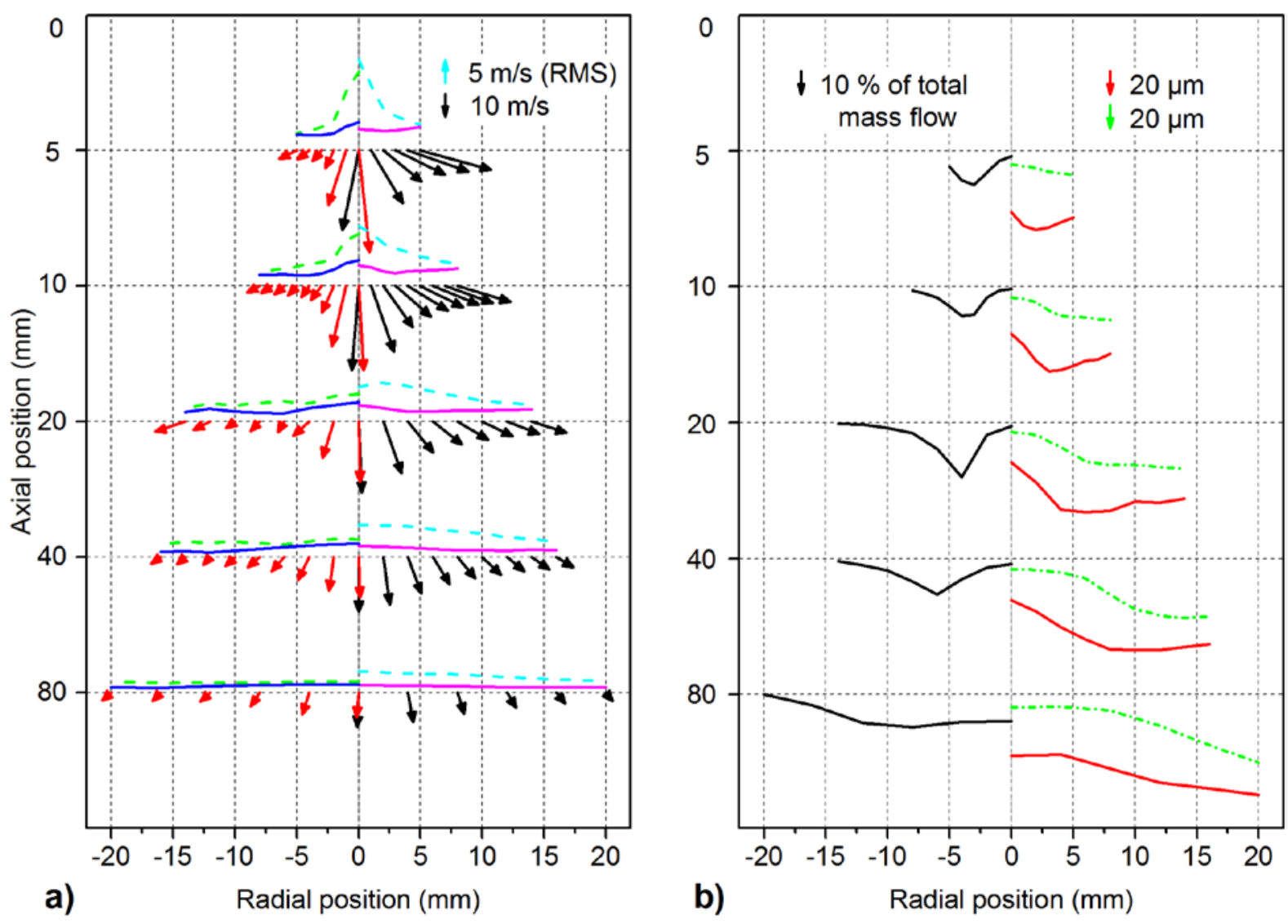

Figure $19 G L R=5 \%$, a) velocity vectors with RMS velocity profiles for both gas and liquid phase, b) liquid mass flow, D10 and D32 profiles. 


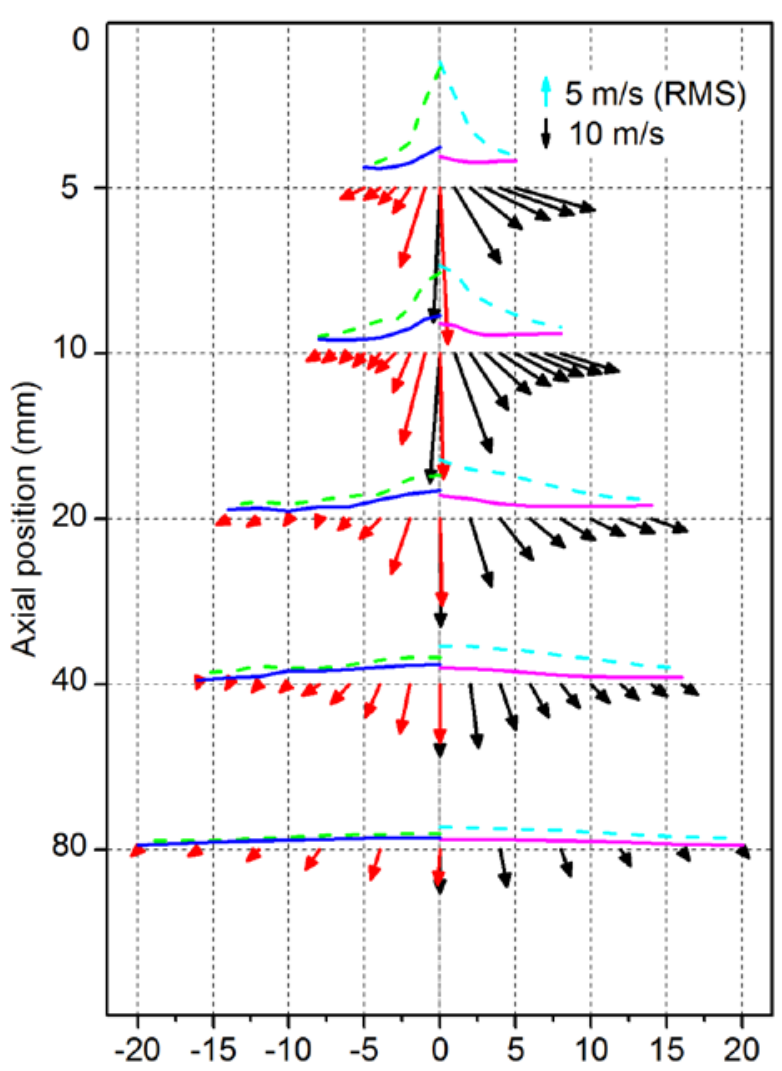

a)

Radial position ( $\mathrm{mm}$ )

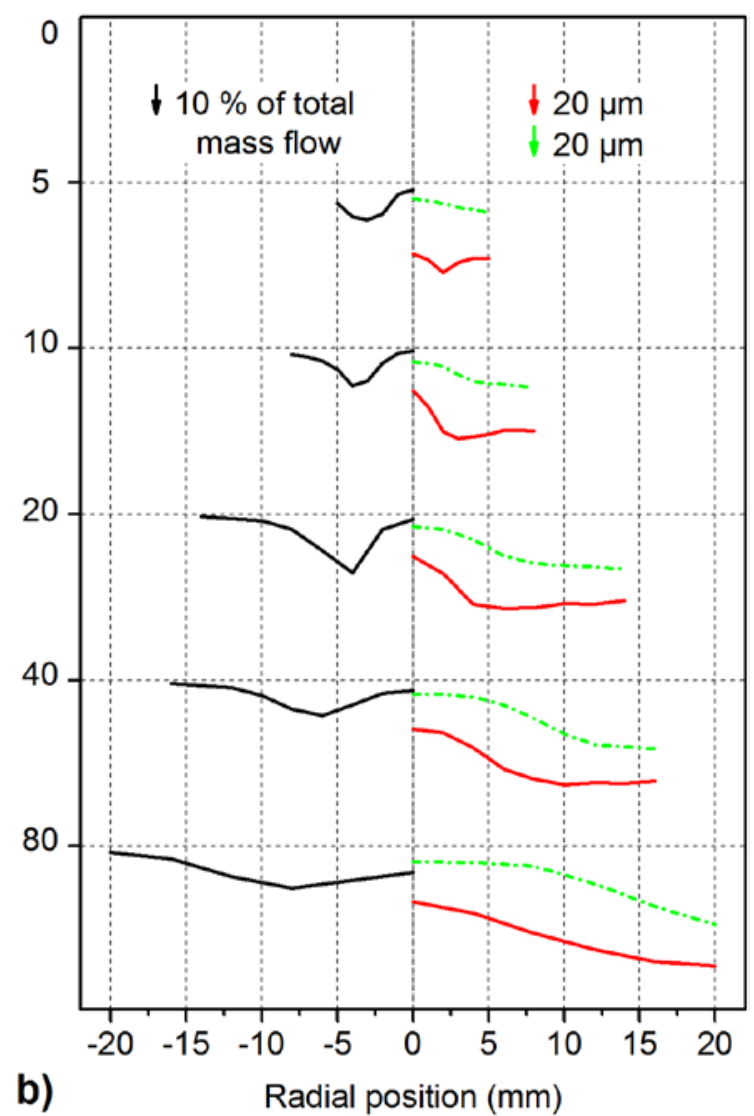

Figure $20 \mathrm{GLR}=10 \%$, a) velocity vectors with RMS velocity profiles for both gas and liquid phase, b) liquid mass flow, D10 and D32 profiles.

\section{Conclusions}

A liquid breakup mechanism based on the effervescent atomization was examined by several approaches. A high-speed back-illumination technique was used with two different configurations to obtain visual information about the liquid deformation and breakup processes. The recordings were then processed by proper orthogonal decomposition (POD) to obtain characteristic shapes of the liquid during the breakup. Moreover, fast Fourier transformation (FFT) was applied to the selected pixels to analyse a frequency spectrum of examined phenomenon. Spray evolution was examined by the phase Doppler analyser (PDA). A final discussion over the results focuses on the information link from different experimental and post-processing techniques based on which several concluding remarks can be made:

- The POD technique enables to minimize the amount of visual data needed for further analysis. It allows a simplification of the problem by examining only the dominant modes and their shapes. The understanding of the next POD modes can be relatively difficult, especially in the case where the POD modes do not have any physical representation. In the examined case, this analysis helped to describe a breakup process in greater detail.

- It was shown that the liquid breakup mechanism can be divided into two separated processes: a) the breakup is caused by radial deformation of the liquid which generates large droplets and ligaments, b) mechanism which occurs in the spray core and is related to the axial gas expansion occurring after the liquid disruption. This process generates small droplets and accelerates them to maximal velocities in the spray. 
Superposition of above mentioned mechanisms is responsible for disintegration of majority of the liquid mass as deducted from mass flow profiles.

- By comparing the curves of ideal gas expansion and Weber number during the bubble expansion it was deducted that the breakup mechanism does not occur at the maximum weber numbers nor the maximum velocity. The breakup starts after the expansion of the gas is finished and the initial disruption of the liquid is caused by thinning of the liquid sheet at the front of the bubble.

- The change in GLR caused an increase of bursting frequency as expected. However, the breakup mechanism seems to be relatively insensitive to GLR. It suggests that the examined atomizer can operate at one breakup mechanism for a broad range of operating conditions, which is partly in contradiction with the previous studies. Nevertheless, it shows a potential for further development of this type of atomizer which is especially suitable for spray viscous fuels at low pressures and low consumption of atomizing gas.

\section{Acknowledgement}

This work has been supported by the project No. GA15-09040S funded by the Czech Science Foundation, the project no. CZ.1.05/2.1.00/19.0397 (NETME Centre TechUp) with the financial support from the Ministry of Education, Youth and Sports of the Czech Republic and and project Reg. no. FSI-S-17-4444 funded by the Brno University of Technology

\section{References}

Ashgriz, N. 2011. Handbook of Atomization and Sprays: Theory and Applications: Springer US.

Berkooz, G., P. Holmes, and J. L. Lumley. 1993. "The proper orthogonal decomposition in the analysis of turbulent flows." Annual Review of Fluid Mechanics 25 (1):539-575.

Buckner, Harry N., and Paul E. Sojka. 1991. "EFFERVESCENT ATOMIZATION OF HIGH-VISCOSITY FLUIDS: PART I. NEWTONIAN LIQUIDS." Atomization and Sprays 1 (3):239-252.

Chen, H., D. L. S. Hung, M. Xu, H. Zhuang, and J. Yang. 2014. "Proper orthogonal decomposition analysis of fuel spray structure variation in a spark-ignition direct-injection optical engine." Experiments in Fluids 55 (4). doi: 10.1007/s00348-014-1703-y.

Chen, Hao, David L. S. Hung, Min Xu, and Jie Zhong. 2013. "ANALYZING THE CYCLE-TO-CYCLE VARIATIONS OF PULSING SPRAY CHARACTERISTICS BY MEANS OF THE PROPER ORTHOGONAL DECOMPOSITION." 23 (7):623-641. doi: 10.1615/AtomizSpr.2013007851.

Chin, J. S. 1995. "Effervescent atomization and internal mixing air-assisted atomization." Int. J. Turbo Jet Engines 12 (2):119-127.

Chin, J. S., and Arthur H. Lefebvre. 1993. "FLOW PATTERNS IN INTERNAL-MIXING, TWIN-FLUID ATOMIZERS." 3 (4):463-475.

Dumouchel, C., J. Cousin, and K. Triballier. 2005. "Experimental analysis of liquid-gas interface at low Weber number: interface length and fractal dimension." Experiments in Fluids 39 (4):651666. doi: 10.1007/s00348-005-1005-5.

Eggers, J., and E. Villermaux. 2008. "Physics of liquid jets." Reports on Progress in Physics 71 (3):79. doi: 10.1088/0034-4885/71/3/036601.

Faeth, G. M., L. P. Hsiang, and P. K. Wu. 1995. "Structure and breakup properties of sprays." International Journal of Multiphase Flow 21 (Suppl):99-127.

Gadgil, Hrishikesh P., and B. N. Raghunandan. 2011. "Some features of spray breakup in effervescent atomizers." Experiments in Fluids 50 (2):329-338. doi: 10.1007/s00348-010-0929-6.

Huang, X., X. Wang, and G. Liao. 2008. "Visualization of two phase flow inside an effervescent atomizer." Journal of Visualization 11 (4):299-308. 
Jedelsky, J, and M Jicha. 2008. "Unsteadiness in effervescent sprays: A new evaluation method and the influence of operational conditions." Atomization and Sprays 18 (1):49-83.

Jedelsky, J, and M Jicha. 2013. "Energy conversion during effervescent atomization." Fuel 111:836844. doi: 10.1016/j.fuel.2013.03.053.

Jedelsky, J., and M. Jicha. 2014. "Droplet dynamics in internally mixed twin-fluid spray." WIT Transactions on Engineering Sciences.

Jedelsky, Jan, and Miroslav Jicha. 2012. "SPATIALLY AND TEMPORALLY RESOLVED DISTRIBUTIONS OF LIQUID IN AN EFFERVESCENT SPRAY." Atomization and Sprays 22 (7):603-626.

Konstantinov, Dancho, Richard Marsh, Phil Bowen, and Andrew Crayford. 2010. "EFFERVESCENT ATOMIZATION FOR INDUSTRIAL ENERGY-TECHNOLOGY REVIEW." Atomization and Sprays 20 (6):525-552. doi: 10.1615/AtomizSpr.v20.i6.40.

Kourmatzis, A., A. Lowe, and A. R. Masri. 2016. "Combined effervescent and airblast atomization of a liquid jet." Experimental Thermal and Fluid Science 75:66-76. doi: http://dx.doi.org/10.1016/j.expthermflusci.2016.02.002.

Lasheras, J. C., E. Villermaux, and E. J. Hopfinger. 1998. "Break-up and atomization of a round water jet by a high-speed annular air jet." Journal of Fluid Mechanics 357:351-379.

Lefebvre, Arthur H. 1988. "A Novel Method of Atomization with Potential Gas Turbine Applications." Defence Science Journal 38 (4):10. doi: 10.14429/dsj.38.5869.

Li, Z. H., Y. X. Wu, C. R. Cai, H. Zhang, Y. L. Gong, K. Takeno, K. Hashiguchi, and J. F. Lu. 2012. "Mixing and atomization characteristics in an internal-mixing twin-fluid atomizer." Fuel 97:306-314. doi: 10.1016/j.fuel.2012.03.006.

Lumley, J. L. 1967. "The Structure of Inhomogeneous Turbulent Flows." In Atmospheric turbulence and radio propagation, edited by A. M. Yaglom and V. I. Tatarski, 166-178. Nauka.

Luong, J. T. K., and P. E. Sojka. 1999. "Unsteadiness in effervescent sprays." Atomization and Sprays 9 (1):87-109.

Marchi, A., J. Nouri, Y. Yan, and C. Arcoumanis. 2010. "Spray stability of outwards opening pintle injectors for stratified direct injection spark ignition engine operation." International Journal of Engine Research 11 (6):413-437. doi: 10.1243/14680874JER605.

Mlkvik, M., P. Stähle, H. P. Schuchmann, V. Gaukel, J. Jedelsky, and M. Jicha. 2015. "Twin-fluid atomization of viscous liquids: The effect of atomizer construction on breakup process, spray stability and droplet size." International Journal of Multiphase Flow 77:19-31. doi: http://dx.doi.org/10.1016/j.ijmultiphaseflow.2015.06.010.

Qian, L. J., and J. Z. Lin. 2011. "Modeling on effervescent atomization: A review." Science ChinaPhysics Mechanics \& Astronomy 54 (12):2109-2129. doi: 10.1007/s11433-011-4536-1.

Rudolf, P., D. Štefan, and R. Klas. 2015. "Spatio-temporal description of the swirling flow in hydraulic turbine draft tube." WasserWirtschaft 105 (1):18-22.

Santangelo, P. J., and P. E. Sojka. 1995. "A holographic investigation of the near-nozzle structure of an effervescent atomizer-produced spray." Atomization and Sprays 5 (2):137-155.

Santolaya, JL, LA Aisa, E Calvo, I Garcia, and JA Garcia. 2010. "Analysis by droplet size classes of the liquid flow structure in a pressure swirl hollow cone spray." Chemical Engineering and Processing 49 (1):125-131. doi: 10.1016/j.cep.2009.12.003.

Sovani, S. D., P. E. Sojka, and A. H. Lefebvre. 2001. "Effervescent atomization." Progress in Energy and Combustion Science 27 (4):483-521.

Stähle, Philipp, Heike P. Schuchmann, and Volker Gaukel. 2015. "Performance and Efficiency of Pressure-Swirl and Twin-Fluid Nozzles Spraying Food Liquids with Varying Viscosity." Journal of Food Process Engineering:n/a-n/a. doi: 10.1111/jfpe.12317.

Sutherland, J. J., P. E. Sojka, and M. W. Plesniak. 1997. "Ligament-controlled effervescent atomization." Atomization and Sprays 7 (4):383-406.

Whitlow, J. D., A. H. Lefebvre, J. R. Rollbuhler, Coal, and Assoc Slurry Technol. 1992. "EXPERIMENTAL STUDIES ON INTERNAL FLOW AND SPRAY CHARACTERISTICS OF EFFERVESCENT ATOMIZERS." Proceedings of the 17th International Conference on Coal Utilization \& Slurry Technologies:171-182. 
Zaremba, Matouš, Lukas Weiß, Milan Malý, Michael Wensing, Jan Jedelský, and Miroslav Jícha. 2017. "Low-pressure twin-fluid atomization: Effect of mixing process on spray formation." International Journal of Multiphase Flow 89:277-289. doi: http://dx.doi.org/10.1016/i.ijmultiphaseflow.2016.10.015. 